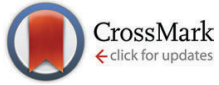

Cite this: Phys. Chem. Chem. Phys., 2014, 16, 24575

\title{
Coherency strain and its effect on ionic conductivity and diffusion in solid electrolytes - an improved model for nanocrystalline thin films and a review of experimental data
}

\author{
C. Korte, ${ }^{\star a}$ J. Keppner, ${ }^{a}$ A. Peters, ${ }^{b}$ N. Schichtel, ${ }^{b}$ H. Aydin ${ }^{b}$ and J. Janek ${ }^{b}$
}

A phenomenological and analytical model for the influence of strain effects on atomic transport in columnar thin films is presented. A model system consisting of two types of crystalline thin films with coherent interfaces is assumed. Biaxial mechanical strain $\varepsilon_{0}$ is caused by lattice misfit of the two phases. The conjoined films consist of columnar crystallites with a small diameter $l$. Strain relaxation by local elastic deformation, parallel to the hetero-interface, is possible along the columnar grain boundaries. The spatial extent $\delta_{0}$ of the strained hetero-interface regions can be calculated, assuming an exponential decay of the deformation-forces. The effect of the strain field on the local ionic transport in a thin film is then calculated by using the thermodynamic relation between (isostatic) pressure and free activation enthalpy $\Delta G^{\#}$. An expression describing the total ionic transport relative to bulk transport of a thin film or a multilayer as a function of the layer thickness is obtained as an integral average over strained and unstrained regions. The expression depends only on known material constants such as Young modulus $Y$, Poisson ratio $\nu$ and activation volume $\Delta V^{\#}$, which can be combined as dimensionless parameters. The model is successfully used to describe own experimental data from conductivity and diffusion studies. In the second part of the paper a comprehensive literature overview of experimental studies on (fast) ion transport in thin films and multilayers along solid-solid hetero-interfaces is presented. By comparing and reviewing the data the observed interface effects can be classified into three groups: (i) transport along interfaces between extrinsic ionic conductors (and insulator), (ii) transport along an open surface of an extrinsic ionic conductor and (iii) transport along interfaces between intrinsic ionic conductors. The observed effects in these groups differ by about five orders of magnitude in a very consistent way. The modified interface transport in group (i) is most probably caused by strain effects, misfit dislocations or disordered transition regions.

Received 11th July 2014,

Accepted 30th September 2014

DOI: $10.1039 / c 4 c p 03055 a$

www.rsc.org/pccp range for stable operation is lower than necessary for sufficiently high ionic conductivity. For anode supported SOFCs with thin electrolyte layers down to $15-20 \mu \mathrm{m}$, the minimum operation temperature is about $650-700{ }^{\circ} \mathrm{C}^{1,2}$ Therefore, concepts for stable solid electrolytes with sufficiently high ionic conductivity at low and intermediate temperatures are required. ${ }^{3}$

\subsection{Strain as an "issue" and as a concept}

Recently, one of these concepts-namely the controlled use of strain, i.e. "strain-based design" of solid electrolytes and their "chemomechanics"-attracts increasing interest. ${ }^{4}$ In some pioneering studies it was shown that strain may positively influence ionic transport. ${ }^{5-8}$ In a number of subsequent experimental, ${ }^{9-15}$ theoretical studies ${ }^{16-23}$ and a few reviews ${ }^{4,21,24-26}$ intrinsic strain as a control parameter has been investigated and even used to tune transport properties in oxide ceramic multilayer microdots. ${ }^{27}$ Mostly, epitaxial strain originating at

\footnotetext{
${ }^{a}$ Institut für Energieforschung, Brennstoffzellen (IEK-3), Forschungszentrum Jülich GmbH, D-52425 Jülich, Germany.E-mail: c.korte@fz-juelich.de

${ }^{b}$ Physikalisch-Chemisches Institut, Justus-Liebig-Universität Gießen,

Heinrich-Buff-Ring 58-62, D-35392 Gießen, Germany
} 
heterophase boundaries was considered, but uniaxially strained single crystals were also studied as well defined model systems. We like to note that strain may often be an intrinsic issue in composite materials for high temperature applications - as different thermal expansion coefficients here always lead to internal strain at heterophase boundaries. ${ }^{28}$ We also like to add that the precise determination of actual strain at an inner interface under operation conditions is difficult, and that the strain state of a specific interface depends crucially on the microstructure of the interface. Thus, a careful experimental design is required in order to avoid incorrect conclusions, as e.g. in the case of electronic contributions of substrates. ${ }^{5}$ As many solid electrolytes are multi-component compounds, segregation effects may also significantly influence the interfacial properties. ${ }^{22,29-33}$

\subsection{Layered structures}

In an attempt to understand the influence of strain on ionic transport along heterophase boundaries on a quantitative basis, we recently performed a systematic experimental study of a series of well-defined layered composite systems along with the presentation of a phenomenological strain-based model: multilayers of YSZ, a ternary fluorite-type oxygen solid electrolyte (YSZ = yttria-stabilized zirconia), with binary rare earth sesquioxides $\left(\mathrm{RE}_{2} \mathrm{O}_{3}, \mathrm{RE}=\mathrm{Y}\right.$, $\mathrm{Lu}, \mathrm{Sc}$ ) allowed the preparation of model-type thin films with defined lattice misfit and corresponding epitaxial strain. ${ }^{6,7,9,12,13}$ The influence of coherency strain and of dislocation networks on the ionic transport was quantitatively analysed in terms of an own theoretical model. It is one of the purposes of the present paper to present an improved model for strain-modified ionic mobility along heterophase boundaries - taking the polycrystalline character and grain boundaries in the thin solid electrolyte films into account.

In the case of space charge based models the focus lies on the specific adsorption of charged point defects in the interface to explain the modified transport coefficients. Due to the specific absorption of one ionic species at the interfaces, a space charge region is formed. In the space charge region the absorbed point defect species is depleted and due to the internal defect equilibria a corresponding point defect species is accumulated, once the Gouy-Chapman model applies. This can result in an increased concentration of mobile charge carriers. The most important experimental studies and theoretical work were reported in the 1980 s and 1990s. ${ }^{34-43}$

However, in (strongly doped) materials like YSZ or CGO (gadolinia doped ceria) with high concentrations of charge carriers, the width of space charge regions is very small. It can be estimated in the order of the Debye length. Thus, the effect on the carrier concentration is negligible. Therefore, we exclude space charge effects from our current treatment. Of course, they should not be neglected in the case of systems with longer Debye lengths. We also exclude-as before-the formation of interphases with a new structure at the core of an interface as well as contribution from segregation effects. We studied the chemical composition of the $\mathrm{YSZ} / \mathrm{RE}_{2} \mathrm{O}_{3}$ interface with high spatial resolution and did not find evidence for segregation or interdiffusion, therefore we consider this assumption as justified. ${ }^{9}$

\subsection{Review}

As a number of interesting theoretical and experimental studies have been published since the first papers on strain-modified ion transport, we also summarise the available data for oxygen solid electrolytes (and fluorine ion conductors) in order to better judge the current state of research. We emphasise that we focus exclusively on studies of strain as a controlling parameter in thin film structures and leave other concepts for the improvement of ionic conductivity (e.g. nanoscaled random microstructure with high density of grain boundaries) aside. However, as grain boundaries in epitaxial nanocrystalline films intrinsically influence the conductivity of these films, their role will also be discussed in Section 4. In essence, the number of systematic experimental studies under well-defined conditions is still comparably small, and further efforts will be required to prove the specific effect of strain on ionic transport in different systems.

\section{Literature survey on experimental studies}

Thin film and multilayer systems, prepared by vapour deposition techniques, have a simple two-dimensional and well-defined geometry, often a uniform texture and thus a uniform interface structure. The measured properties can be easily attributed to a distinct interface type, which makes thin film systems suitable for interface transport studies. Most of the thin films studies in the literature were performed on $\mathrm{O}^{2-}$-conductors, ${ }^{5-15,24,44-51}$ but also $\mathrm{F}^{-}, \mathrm{Li}^{+}, \mathrm{H}^{+}$and $\mathrm{Ag}^{+}$ion conducting solid electrolytes have been investigated. ${ }^{52-59}$

Two recent reviews on chemomechanic effects, electrocatalysis and diffusion in ceramic materials, provide overviews of about 14 experimental and some theoretical studies of ionic conductivity of strained interfaces. ${ }^{4,21}$ The maximum observed or predicted increase of the ionic conductivity relative to the bulk value is discussed. Expanding this reviews, we have reviewed and listed 24 experimental studies from other groups and own studies in Table 1, which were published in the last decade. The literature survey is restricted on anion conductors to maintain a better mutual comparability $\left(\mathrm{O}^{2-}\right.$ and $\left.\mathrm{F}^{-}\right) .{ }^{5-15,24,44-51,53,55,57,59}$

\subsection{Data evaluation}

The conductivity and diffusivity data of thin film and multilayer studies with different layer thicknesses cannot be compared directly. Comparing the value for the bulk transport with the highest measured values for thin film samples, as it is reported in an experimental study, can be misleading. The measured (total) conductivity $\sigma_{\text {tot }}$ or diffusivity $D_{\text {tot }}$ of a sample depends essentially on the layer thickness $d$. In the simple case of independent and parallel transport through the bulk phase(s) of the ionic conducting film(s) and along the phase boundaries the following expression can be derived: ${ }^{6,48}$

$$
\frac{\sigma_{\mathrm{tot}}(d)}{\sigma_{\mathrm{vol}}}=1+\left(\frac{\sigma_{\mathrm{int}}}{\sigma_{\mathrm{vol}}}-1\right) \frac{2 \delta_{0}{ }^{\prime}}{d} \quad \text { if } \quad d>2 \delta_{0}{ }^{\prime}
$$




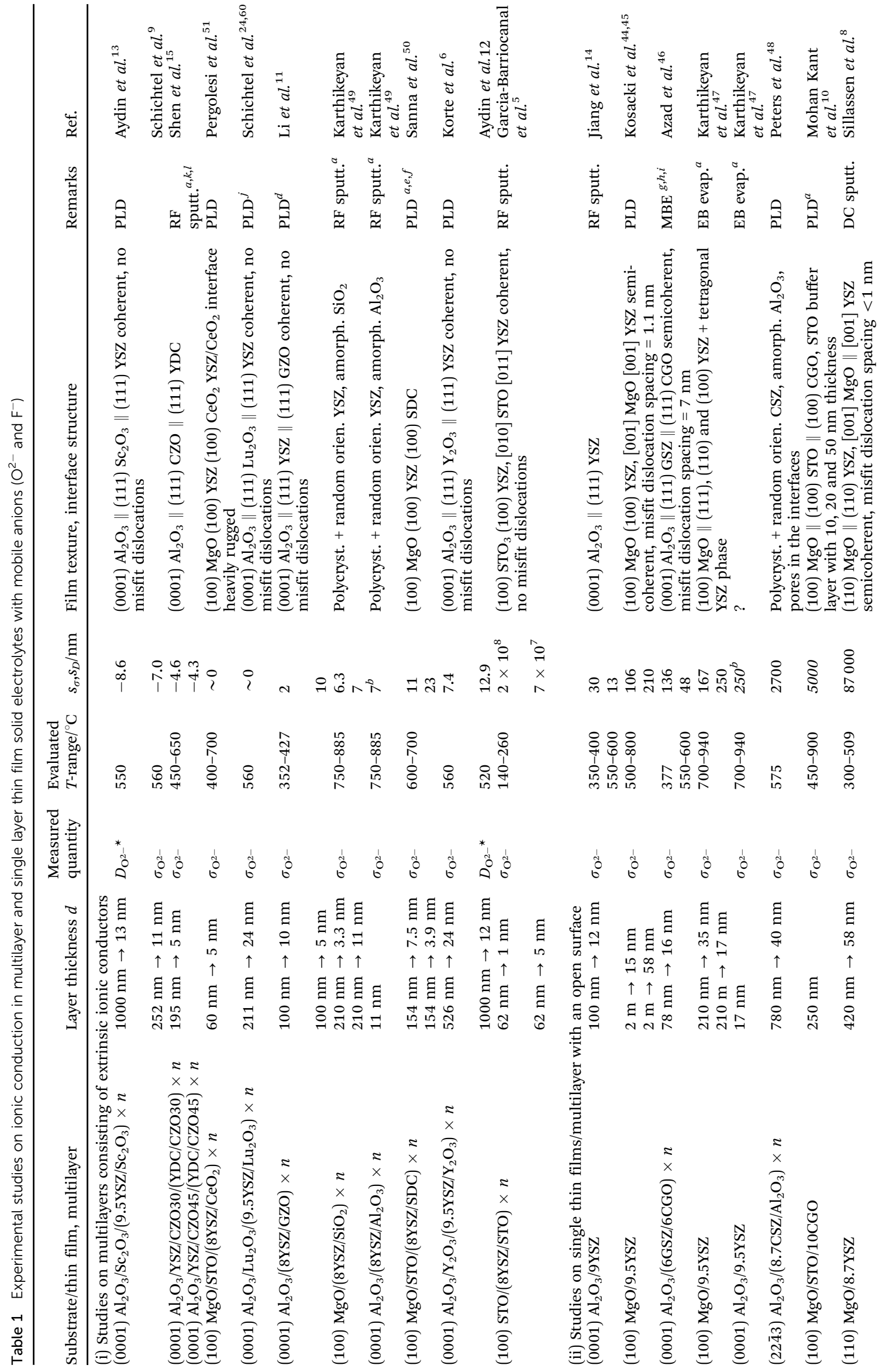




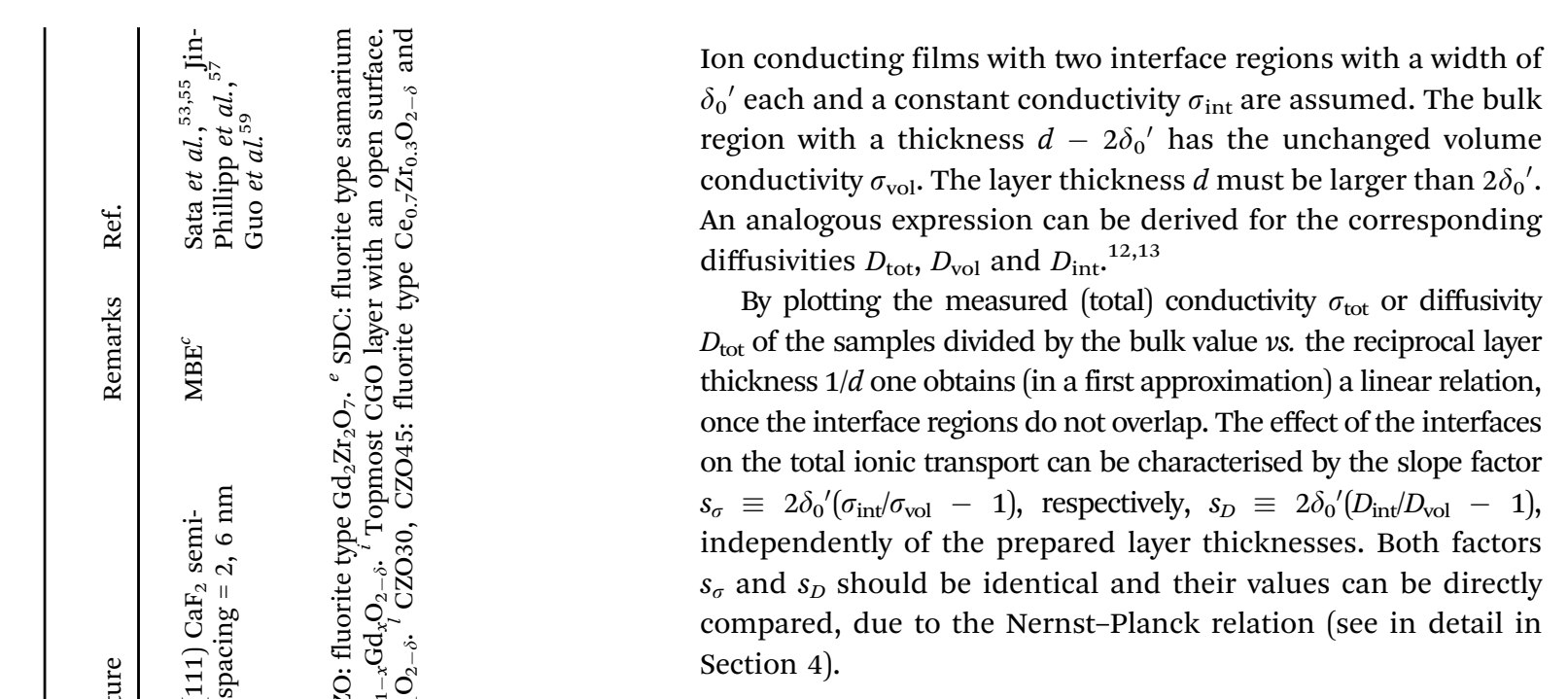

\subsection{Classification of the experimental data from the literature}

The magnitudes of the slope factors $s_{\sigma}$ and $s_{D}$ in Table 1 evaluated from the published data differ by about eight orders of magnitude. Because of a non-linear behaviour, the slope factors for some of the studies vary considerably when including the data for the thinnest investigated layer thicknesses. In this case we specify two values, together with the evaluated layer thickness range. In the case of studies where only one measurement for a single thickness is given, the slope factor is italicised.

Considering the material properties of the used ionic conductors, the morphology and the structure of the heterointerfaces we classify all studied systems into three groups according to the magnitude of the slope factors:

(i) Strain control: multilayers consisting of extrinsic ionic conductors (and insulators). ${ }^{5,6,9,11-13,24,49-51}$ The slope factors have a value of about $10^{1} \mathrm{~nm}$ at maximum.

(ii) Surface control: single thin films/multilayers consisting of an extrinsic ionic conductor, exhibiting an open surface. $8,10,14,44-48$ The value of the slope factors is above $10^{1} \mathrm{~nm}$ and about $10^{5} \mathrm{~nm}$ at maximum.

(iii) Space charge control: multilayers consisting of intrinsic ionic conductors. ${ }^{53,55,57,59}$ A slope factor in the order of $10^{3} \mathrm{~nm}$ can be observed.

There is clear evidence that the highest increase of the ionic conductivity observed in the study of Garcia et al. ${ }^{5}$ of up to eight orders of magnitude in ultra-thin and strained YSZ films is rather due to effects on electronic conduction than to an increased ionic mobility. ${ }^{61-63}$ Comparing the reported sample properties and experimental conditions, it is possible to attribute the observed interface effects in each group to a distinct (most probable) mechanism.

Space charge regions as an origin for increased interface transport can be attributed to group (iii) for systems with intrinsic ionic conductors. A slope factor in the order of $10^{3} \mathrm{~nm}$ can be found. These considerably high values result from the low conductivity of intrinsic bulk materials and a relatively strong conductivity increase in the space charge region. Thus, even with this increase the total conductivity 
$\sigma_{\text {tot }}$ remains low compared to the bulk conductivity of extrinsic ionic conductors.

In group (i) for systems with extrinsic ionic conductors the smallest effects on the interface transport appear. The (absolute) values of the slope factors are only in the order of $10^{1} \mathrm{~nm}$. Because of the absence of space charge regions, only strain fields, misfit dislocations or disordered transition regions in solid-solid phase boundaries may cause these moderate effects. The grain boundary density may easily have a conductivity effect in the same order of magnitude, however, often reducing the conductivity. Thus, strain effects may then not increase the conductivity relative to single crystalline material, but may help to reduce the negative effect of blocking grain boundaries. We emphasise that the influence of grain boundaries depends on the size of the film crystallites. Unfortunately, information on the film microstructure is not given in all cited references.

In group (ii) also systems with extrinsic ionic conductors can be found, but with slope factors above $10^{1} \mathrm{~nm}$ and at maximum up to $10^{5} \mathrm{~nm}$. This can be explained by open surfaces of the ion conducting phases as a common feature of all samples. Atomic transport along surfaces (solid-gas interfaces) is usually much faster compared to grain or phase boundary transport (solidsolid interfaces). The decreased number of bonds of a surface atom and reconstruction phenomena will considerably change the activation energy for jump processes. ${ }^{64}$ For the majority of the experimental studies attributed to this group a slope factor in the order of $10^{2} \mathrm{~nm}$ can be found, i.e. one order of magnitude higher compared to group (i).

In the following, we will perform an analytical treatment of the elastic strain effects occurring in category (i) on a phenomenological basis using linear transport theory. Aim is an analytical expression for the ionic conductivity or the diffusion coefficient of interfacially strained nano-/microscaled thin films, consisting of ionic conducting and insulating phases. A correlation between the transport parameters and the dimensions of the ionic conducting layers and the mutual mismatch between the adjacent phases are derived and compared with available experimental data. A simplified treatment has already been published by us in previous papers. ${ }^{9,24}$ Major improvement of our model is the introduction of a grain boundary dependent strain relaxation in the solid electrolyte films, describing the thin films in a more realistic way.

\section{Phenomenological model for strain effects}

\subsection{Lattice mismatch and elastic strain}

A thin film (multilayer) model system is considered. It is assumed that the adjacent layers consist of two crystalline phases, 1 and 2. Both thin film materials are elastically deformable. There is a lattice mismatch due to different lattice constants but the interfaces are still coherent:

$$
f_{1 / 2}=\frac{d_{h k l, 2}-d_{h k l, 1}}{d_{h k l, 1}} \quad \text { and } \quad f_{2 / 1}=\frac{d_{h k l, 1}-d_{h k l, 2}}{d_{h k l, 2}}
$$

Hereby, $f_{1 / 2}$ is the mismatch of phase 1 relative to phase 2 and $f_{2 / 1}$ the mismatch of 2 relative to 1 . The mismatch between phase 1 and 2 is assumed to be small, typically below $\pm 5 \%$. In mechanical equilibrium a common lattice distance $d_{h k l, 0}$ in the interface plane is reached, if both phases are elastically deformable:

$$
\begin{aligned}
f_{1 / 2} \approx-f_{2 / 1} & \approx \frac{d_{h k l, 0}-d_{h k l, 1}}{d_{h k l, 1}}-\frac{d_{h k l, 0}-d_{h k l, 2}}{d_{h k l, 2}} \\
& \approx \varepsilon_{0,1}-\varepsilon_{0,2}
\end{aligned}
$$

\subsection{Elastic strains and stresses}

The thin films of the ionic conducting phase consist of columnar crystallites. As depicted in Fig. 1, the direction perpendicular to the interface planes is defined as the $z$-axis and parallel to it as $x$ - and $y$-axes. For the further considerations the phase indices 1 and 2 are dropped. Each crystallite has two phase boundary

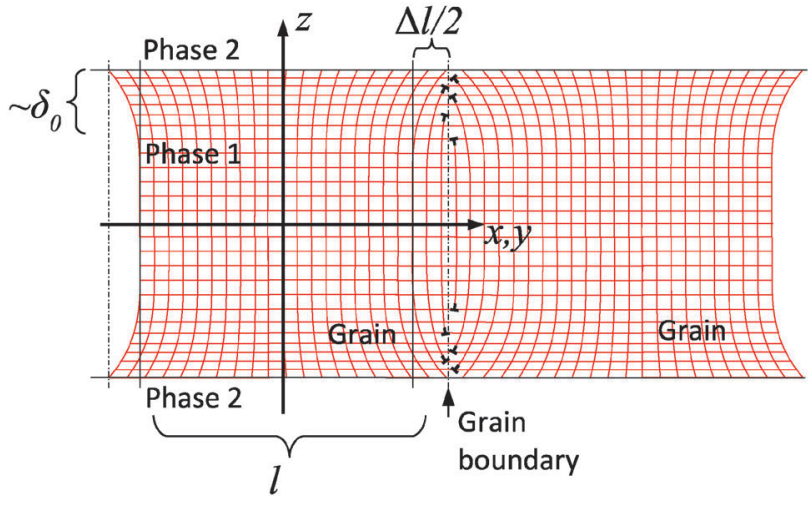

a) tensile strain, $f_{1 / 2}>0$

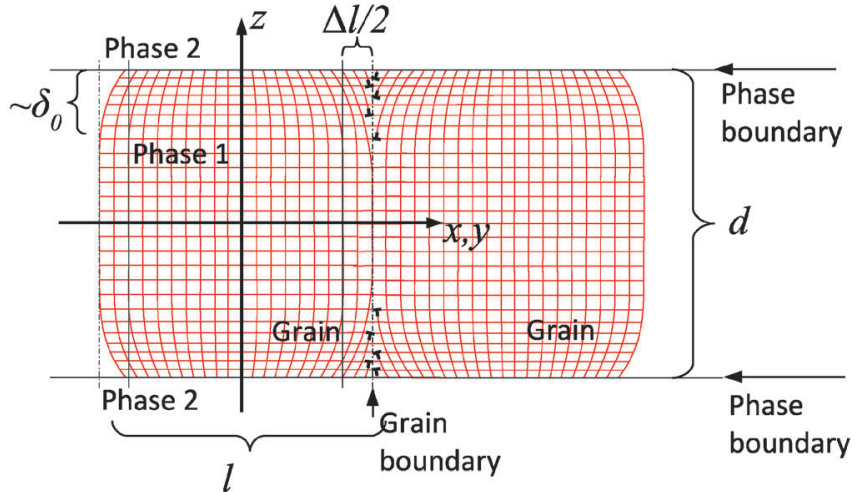

b) compressive strain, $f_{1 / 2}<0$

Fig. 1 Elastically deformable crystallites of phase 1 with a diameter 1 and a height $d$ (layer thickness). The tensile strained case is shown in (a) and the compressively strained case in (b). The lattice planes are matched across the interface to the crystallites of phase 2 (not shown). Elastic strain relaxation by local deformation took place in the vicinity of both interfaces by introduction of dislocations in the grain boundaries. The spatial extent of the strained interface regions is proportional to $\delta_{0}$. 
planes at $z=-d / 2$ and $z=d / 2$, i.e. a height $d$ (= layer thickness). A biaxial strain state is assumed. The strain due to the mismatch is only applied at the phase boundaries in the $x-y$-plane. The diameter of the crystallites in the phase boundary planes in $x$ - and $y$-directions has the width $l$. The elastic properties in both phases are assumed to be similar. The interface strain will be divided approximately in equal parts by both adjacent phases:

$$
\begin{gathered}
\varepsilon_{0,1} \approx-\varepsilon_{0,2} \\
f_{1 / 2} \approx-f_{2 / 1} \approx 2 \varepsilon_{0,1}=-2 \varepsilon_{0,2}=2 \varepsilon_{0}
\end{gathered}
$$

If the diameter of adjacent crystallites is relatively small, the interface strain in both phases can be relaxed by local deformation in regions close to the interfaces. Thus, in each crystallite two interface regions with a spatially decaying strain are formed. To obtain the function describing the decay of the elastic deformation one has to solve an integral equation, which connects the elastic deformation, respectively, the resulting strain and stress with the total elastic energy of the system. The desired function is obtained by minimising the total elastic energy of the system, i.e. for mechanical equilibrium.

As a simple approximation an exponential decay for the elastic deformation can be assumed. Murakami et al., Luryi et al., Stoica et al. and Fischer et al. also apply exponential strain relaxation for the description of elastic strain states in epitaxially grown and island shaped crystallites on silicon substrates. ${ }^{65-69}$ Due to this, the thickness limit for the formation of coherent interfaces is increased. ${ }^{64,70}$ The volume elements in each layer may be elastically shifted only parallel to the $x$-and the $y$-axis, see Fig. 1. To compensate the interface mismatch by an elastic deformation $\pm l \varepsilon_{0} / 2$ the shifts $u_{x}$ and $u_{y}$ will increase linearly with the distance from the $x$ - $z$-plane, respectively, the $y$ - $z$-plane. For $x=0$ the shift in the $x$-direction is zero and for $y=0$ the shift in the $y$-direction is zero. The maximum shift for a volume element in a layer is reached in the phase boundary planes at $z=-d / 2$ and $z=d / 2$ at the outer limits $x= \pm l / 2$ and $y= \pm l / 2$ of the crystallite. The shift $u_{z}$ in the $z$-direction depends only on the distance from the $x-y$-plane.

The strain fields originating from the phase boundary planes will superpose, if the height $d$ of the crystallite is small. The resulting elastic deformations at the phase boundary planes at $z=-d / 2$ and $z=d / 2$ cannot exceed the deformation $x \varepsilon_{0} / 2$ required to compensate the interface mismatch. For an exponential decay of the elastic deformation, these conditions are satisfied by the following functions $\left(\propto \mathrm{e}^{z / \delta_{0}-d / 2 \delta_{0}}, \propto \mathrm{e}^{-z / \delta_{0}-d / 2 \delta_{0}}\right.$, scaling factor of $\left.1+\mathrm{e}^{-d / \delta_{0}}\right)$ :

$$
\begin{aligned}
u_{x} & =\frac{\varepsilon_{0} x}{1+\mathrm{e}^{-d / \delta_{0}}}\left(\mathrm{e}^{\frac{z-d / 2}{\delta_{0}}}+\mathrm{e}^{-\frac{z+d / 2}{\delta_{0}}}\right) \\
& =\varepsilon_{0} x \frac{\cosh z / \delta_{0}}{\cosh \frac{d}{2 \delta_{0}}} \\
u_{y} & =\varepsilon_{0} y \frac{\cosh z / \delta_{0}}{\cosh \frac{d}{2 \delta_{0}}}
\end{aligned}
$$

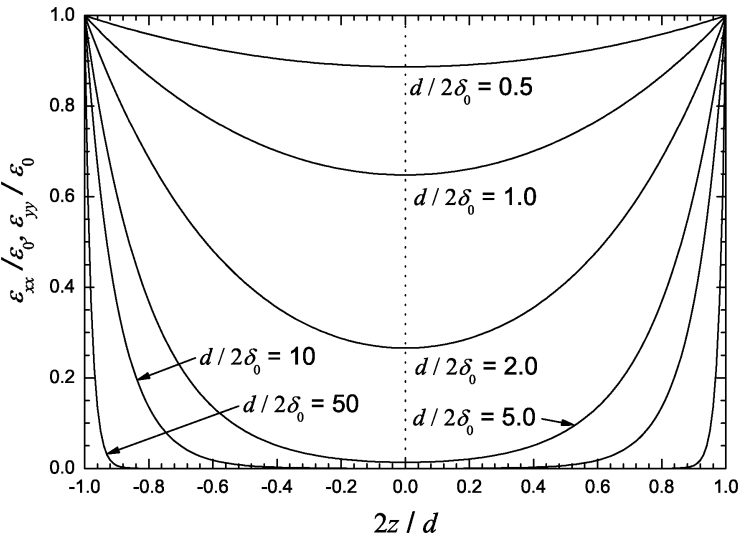

Fig. 2 Profile of the relative biaxial strains $\varepsilon_{x x} / \varepsilon_{0}$ and $\varepsilon_{y y} / \varepsilon_{0} v s$. the relative position $2 z / d$. The phase boundaries are situated at $2 z / d=-1$ and 1 . Curves are plotted for different ratios $d / 2 \delta_{0}$ between layer thickness and the extent of the strained interface region. Noticeable overlap of the strain fields appears, if $d / 2 \delta_{0}<5$.

$$
u_{z}=u_{z}(z)
$$

The parameter $\delta_{0}$ describes the width of the strained interface region (decay of the deformation by a factor of $1 / e$ ). From the elastic deformations in eqn (5) to (7) the strain components $\varepsilon_{x x}, \varepsilon_{y y}, \varepsilon_{z y}, \varepsilon_{z x}$ and $\varepsilon_{x y}$ can be calculated: $\dagger$

$$
\begin{gathered}
\varepsilon_{x x}=\varepsilon_{y y}=\varepsilon_{0} \frac{\cosh z / \delta_{0}}{\cosh \frac{d}{2 \delta_{0}}} \\
\varepsilon_{y z}=\frac{\varepsilon_{0} y}{2 \delta_{0}} \frac{\sinh z / \delta_{0}}{\cosh \frac{d}{2 \delta_{0}}} \\
\varepsilon_{x z}=\frac{\varepsilon_{0} x}{2 \delta_{0}} \frac{\sinh z / \delta_{0}}{\cosh \frac{d}{2 \delta_{0}}} \\
\varepsilon_{x y}=0
\end{gathered}
$$

In Fig. 2 the profile of the biaxial strain $\varepsilon_{x x}$ and $\varepsilon_{y y}$ is depicted $v s$. the position on the $z$-axis. As a boundary condition,

$\dagger$ The components of the Cauchy strain tensor $\varepsilon$ can be calculated from the elastic deformations:

$$
\begin{aligned}
\boldsymbol{\varepsilon} & =\left(\begin{array}{ccc}
\varepsilon_{x x} & \varepsilon_{x y} & \varepsilon_{x z} \\
\varepsilon_{y x} & \varepsilon_{y y} & \varepsilon_{y z} \\
\varepsilon_{z x} & \varepsilon_{z y} & \varepsilon_{z z}
\end{array}\right) \\
& =\left(\begin{array}{ccc}
\frac{\partial u_{x}}{\partial x} & \frac{1}{2}\left(\frac{\partial u_{x}}{\partial y}+\frac{\partial u_{y}}{\partial x}\right) & \frac{1}{2}\left(\frac{\partial u_{x}}{\partial z}+\frac{\partial u_{z}}{\partial x}\right) \\
\frac{1}{2}\left(\frac{\partial u_{y}}{\partial x}+\frac{\partial u_{x}}{\partial y}\right) & \frac{\partial u_{y}}{\partial y} & \frac{1}{2}\left(\frac{\partial u_{y}}{\partial z}+\frac{\partial u_{z}}{\partial y}\right) \\
\frac{1}{2}\left(\frac{\partial u_{z}}{\partial x}+\frac{\partial u_{x}}{\partial z}\right) & \frac{1}{2}\left(\frac{\partial u_{z}}{\partial y}+\frac{\partial u_{y}}{\partial z}\right) & \frac{\partial u_{z}}{\partial z}
\end{array}\right)
\end{aligned}
$$


there are no (external) forces perpendicular to the phase boundary planes, i.e. the stress component $\sigma_{z z}$ is zero:

$$
\sigma_{z z}=0
$$

The stress components $\sigma_{x x}, \sigma_{y y}, \sigma_{y z}, \sigma_{x z}$ and $\sigma_{x y}$ as well as the strain component $\varepsilon_{z z}$ are obtained from eqn (9) to (13) using the generalised Hooke’s law: $\$$

$$
\begin{aligned}
\sigma_{x x} & =\sigma_{y y}=\frac{Y}{1-\nu} \varepsilon_{0} \frac{\cosh z / \delta_{0}}{\cosh \frac{d}{2 \delta_{0}}} \\
\sigma_{y z} & =\frac{Y}{1+\nu} \frac{\varepsilon_{0} y}{2 \delta_{0}} \frac{\sinh z / \delta_{0}}{\cosh \frac{d}{2 \delta_{0}}} \\
\sigma_{x z} & =\frac{Y}{1+\nu} \frac{\varepsilon_{0} x}{2 \delta_{0}} \frac{\sinh z / \delta_{0}}{\cosh \frac{d}{2 \delta_{0}}} \\
\sigma_{x y} & =0 \\
\varepsilon_{z z} & =-\frac{2 \nu}{1-\nu} \varepsilon_{0} \frac{\cosh z / \delta_{0}}{\cosh \frac{d}{2 \delta_{0}}}
\end{aligned}
$$

For the sake of simplicity of the theoretical treatment, an isotropic system is assumed, which can be described only by two elastic constants, Young's modulus $Y$ and the Poisson ratio $\nu$. The system has ideal elastic properties, i.e. the interface strain leads only to elastic distortion of the interface regions. We ignore more complex effects of mechanically induced lattice transformation or changes in the short-range order of ions. ${ }^{71}$

\subsection{Total elastic energy, width of the strained interface regions}

The value of the parameter $\delta_{0}$, describing the spatial extent of the strained region in mechanical equilibrium, can be gained by minimising the total elastic energy $E_{\text {elast }}$ of the system:

$$
E_{\text {elast }}=\frac{1}{2} \int_{V} \tilde{\boldsymbol{\sigma}} \cdot \tilde{\boldsymbol{\varepsilon}} \mathrm{d} V=\frac{1}{2} \int_{V} \sum_{i, j} \sigma_{i j} \varepsilon_{i j} \mathrm{~d} V
$$

\$ The strain components $\tilde{\boldsymbol{\varepsilon}}=\left(\varepsilon_{x x}, \varepsilon_{y y}, \varepsilon_{z z}, 2 \varepsilon_{y z}, 2 \varepsilon_{x z}, 2 \varepsilon_{x y}\right)$ and the stress components $\tilde{\boldsymbol{\sigma}}=\left(\sigma_{x x}, \sigma_{y y}, \sigma_{z z}, \sigma_{y z}, \sigma_{x z}, \sigma_{x y}\right)$ in an isotropic system are connected by the compliance tensor (using Voight notation):

$$
\tilde{\boldsymbol{\varepsilon}}=\frac{1}{Y}\left(\begin{array}{cccccc}
1 & -\nu & -\nu & 0 & 0 & 0 \\
-\nu & 1 & -\nu & 0 & 0 & 0 \\
-\nu & -\nu & 1 & 0 & 0 & 0 \\
0 & 0 & 0 & 2(1+\nu) & 0 & 0 \\
0 & 0 & 0 & 0 & 2(1+\nu) & 0 \\
0 & 0 & 0 & 0 & 0 & 2(1+\nu)
\end{array}\right) \tilde{\boldsymbol{\sigma}}
$$

Inserting all strain and stress components from eqn (9) to (19) in eqn (20) and performing an integration within the boundaries from $x=-l / 2 \ldots l / 2, y=-l / 2 \ldots l / 2$ and $z=-d / 2 \ldots d / 2$ one gets:

$$
\begin{aligned}
E_{\text {elast }}= & \frac{Y}{1-\nu} \varepsilon_{0}^{2} \frac{l^{2} d}{2}\left[\frac{2 \delta_{0}}{d} \tanh \frac{d}{2 \delta_{0}}\left[1+\frac{2}{3} \frac{1-\nu}{1+\nu}\left(\frac{l}{4 \delta_{0}}\right)^{2}\right]\right. \\
& \left.+\frac{1}{\cosh ^{2} \frac{d}{2 \delta_{0}}}\left[1-\frac{2}{3} \frac{1-\nu}{1+\nu}\left(\frac{l}{4 \delta_{0}}\right)^{2}\right]\right]
\end{aligned}
$$

The minimum of the elastic energy $E_{\text {elast }}$ can be found by derivation of eqn (21) with respect to $\delta_{0}$ and setting equal to zero. Only one solution is physically meaningful:

$$
\delta_{0}=\frac{1}{4} \sqrt{\frac{2}{3} \frac{1-\nu}{1+\nu}} \cdot l
$$

Within the limits of this model the width $\delta_{0}$ of the elastically strained interface regions depends directly on the diameter $l$ of the crystallites. Typical values for the Poisson ratio $\nu$ of ceramic materials are in the range between 0.3 and 0.4 , i.e. $\delta_{0}$ is about $13 \%$ to $15 \%$ of the diameter $l$ of the crystallites.

\subsection{Local (isotropic) pressure}

The corresponding local isotropic pressure $p$ can be calculated from the stress components $\sigma_{x x}, \sigma_{y y}$ and $\sigma_{z z}$ in eqn (15) to (13): $\S$

$$
p(z)=-\frac{2}{3} \frac{Y}{1-\nu} \varepsilon_{0} \frac{\cosh z / \delta_{0}}{\cosh \frac{d}{2 \delta_{0}}}
$$

At the phase boundary planes, $z= \pm d / 2$, the local isotropic pressure reaches its maximum value:

$$
p( \pm d / 2)=-\frac{2}{3} \frac{Y}{1-\nu} \varepsilon_{0}
$$

\subsection{Local ionic conductivity}

The diffusion coefficient $D$ and the ionic conductivity $\sigma$ of $\mathrm{O}^{2-}$-ions are pressure dependent. For simplification, we focus on extrinsic ionic conductors with oxygen vacancies $\mathrm{V}_{\mathrm{O}}^{\bullet \bullet}$ as the predominant mobile defect. Assuming an activated jump process one obtains according to the transition state theory:

$$
\begin{aligned}
& \sigma=\sigma_{\mathrm{V}_{\mathrm{O}}^{\bullet}}=\frac{(2 F)^{2}}{R T} c_{\mathrm{V}_{\mathrm{O}}^{\bullet}} \beta \lambda^{2} \omega_{0} \mathrm{e}^{-\frac{\Delta G^{\#}}{R T}} \\
& D=\frac{c_{\mathrm{V}_{\mathrm{O}}^{\bullet}}}{c} D_{\mathrm{V}_{\mathrm{O}}}=\frac{c_{\mathrm{V}_{\mathrm{O}}^{\bullet}}}{c} \beta \lambda^{2} \omega_{0} \mathrm{e}^{-\frac{\Delta G^{\#}}{R T}}
\end{aligned}
$$

$\S$ The isotropic pressure is determined by the trace of the stress tensor $\sigma$ :

$$
p=-\frac{1}{3}\left(\sigma_{x x}+\sigma_{y y}+\sigma_{z z}\right)
$$




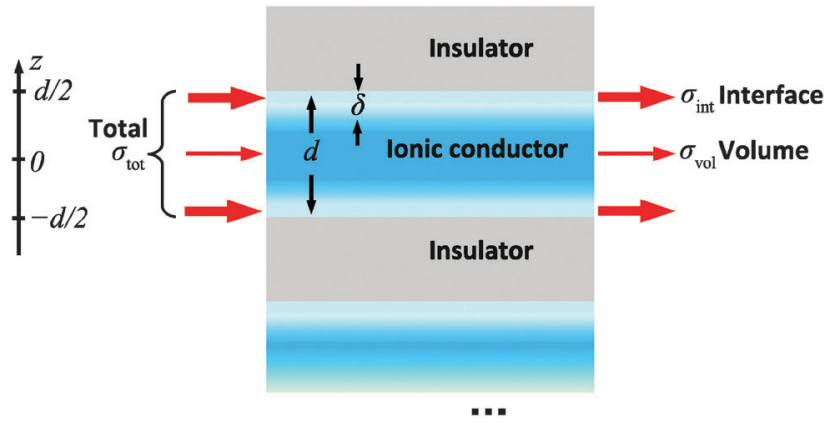

Fig. 3 Parallel conduction $\sigma_{\mathrm{vol}}$ in the volume (bulk) and along interface regions close to the phase boundary with a modified conductivity $\sigma_{\text {int }}$

If we can neglect the pressure dependence of local point defect equilibria on the vacancy concentration $c_{\mathrm{V}_{0}}$, the differentiation of eqn (26) in respect to the pressure $p$ yields:

$$
\left(\frac{\partial \ln \sigma}{\partial p}\right)_{T}=-\frac{\Delta V^{\#}}{R T} \text { and }\left(\frac{\partial \ln D}{\partial p}\right)_{T}=-\frac{\Delta V^{\#}}{R T}
$$

The pressure dependence of the geometric factor $\beta$, the jump distance $\lambda$, the concentration $c$ of oxide ions and the Debye frequency $\left(\sim \omega_{0}\right)$ is also not taken into account. ${ }^{72}$ In this case the pressure induced change of transport coefficients in eqn (27) depends only on the activation volume $\Delta V^{\#}$ of the migrating point defect.

As depicted in Fig. 3 only ionic transport parallel to the phase boundaries will be considered. The value of the ionic conductivity for the unstrained volume phase (for $p=0$ ) will be denoted $\sigma_{\mathrm{vol}}$ and the value of the diffusion coefficient of the unstrained volume phase $D_{\mathrm{vol}}$. To obtain an expression for the local ionic conductivity $\sigma(z)$ as a function of the position $z$ perpendicular to the phase boundary planes, eqn (27) has to be integrated within the limits $\sigma_{\mathrm{vol}} \ldots \sigma(z)$ and $0 \ldots p(z)$. The same applies to obtain an expression for the local diffusion coefficient $D(z)$ :

$$
\ln \frac{\sigma(z)}{\sigma_{\mathrm{vol}}}=-\frac{\Delta V^{\#}}{R T} p(z) \quad \text { and } \quad \ln \frac{D(z)}{D_{\mathrm{vol}}}=-\frac{\Delta V^{\#}}{R T} p(z)
$$

As long as only relative values in relation to the unstrained bulk are considered, the expressions to treat ionic conductivity and the diffusion coefficient have an identical form. Thus, in the following only the expression for the ionic conductivity will be shown for brevity. By inserting the local pressure $p(z)$ from eqn (28) into eqn (24) one yields:

$$
\ln \frac{\sigma(z)}{\sigma_{\mathrm{vol}}}=\alpha \varepsilon_{0} \frac{\cosh z / \delta_{0}}{\cosh \frac{d}{2 \delta_{0}}} \quad \text { with } \quad \alpha=\frac{2}{3} \frac{\Delta V^{\#}}{R T} \frac{Y}{1-\nu}
$$

The dimensionless parameter $\alpha$ combines all material constants, as Young's modulus $Y$, the Poisson ratio $\nu$ and the activation volume $\Delta V^{\#}$ for the atomic jumps.
3.6 The total conductivity as an average between strained and unstrained regions

The total conductivity $\sigma_{\text {tot }}$ perpendicular to the $z$-axis can be calculated as an integral average of the local conductivity $\sigma(z)$ within the limits $z=-d / 2 \ldots d / 2$ (see Fig. 3):

$$
\frac{\sigma_{\mathrm{tot}}(d)}{\sigma_{\mathrm{vol}}}=\frac{1}{d} \int_{-d / 2}^{d / 2} \sigma(z) \mathrm{d} z=\frac{2}{d} \int_{0}^{d / 2} \sigma(z) \mathrm{d} z
$$

By inserting eqn (29) in eqn (30) an expression for the total conductivity $\sigma_{\text {tot }}$ relative to the volume conductivity $\sigma_{\mathrm{vol}}$ of a thin film with the thickness $d$ can be derived:

$$
\frac{\sigma_{\mathrm{tot}}(d)}{\sigma_{\mathrm{vol}}}=\frac{2}{d} \int_{0}^{d / 2} \exp \left(\alpha \varepsilon_{0} \frac{\cosh z / \delta_{0}}{\cosh \frac{d}{2 \delta_{0}}}\right) \mathrm{d} z
$$

By introducing a dimensionless film thickness parameter $d^{*}=d / 2 \delta_{0}$ and a new integration variable $z^{\prime}=z / \delta_{0}$ the integral in eqn (31) can be simplified:

$$
\begin{aligned}
\frac{\sigma_{\mathrm{tot}}\left(d^{*}\right)}{\sigma_{\mathrm{vol}}}=\frac{1}{d^{*}} \int_{0}^{d^{*}} \exp \left(\alpha \varepsilon_{0} \frac{\cosh z^{\prime}}{\cosh d^{*}}\right) \mathrm{d} z^{\prime} \\
\text { with } \quad d^{*}=\frac{d}{2 \delta_{0}} \quad \text { and } \quad \alpha=\frac{2}{3} \frac{\Delta V^{\#}}{R T} \frac{Y}{1-\nu}
\end{aligned}
$$

To obtain analogue expressions for the diffusion coefficient, in eqn (32) and all following equations only $\sigma_{\mathrm{tot}}\left(d^{*}\right) / \sigma_{\mathrm{vol}}$ has to be substituted with $D_{\text {tot }}\left(d^{*}\right) / D_{\text {vol }}$.

Unfortunately, there is no analytical solution for integrals of the type $\int \exp [a \cosh (t)] \mathrm{d} t$. In Fig. 4 numerically calculated values for the relative ionic conductivity $\sigma_{\text {tot }} / \sigma_{\mathrm{vol}}$ are plotted $v s$. the reciprocal film thickness parameter $1 / d^{*}$, using typical values of $\alpha \varepsilon_{0}$ for ceramic thin film systems.

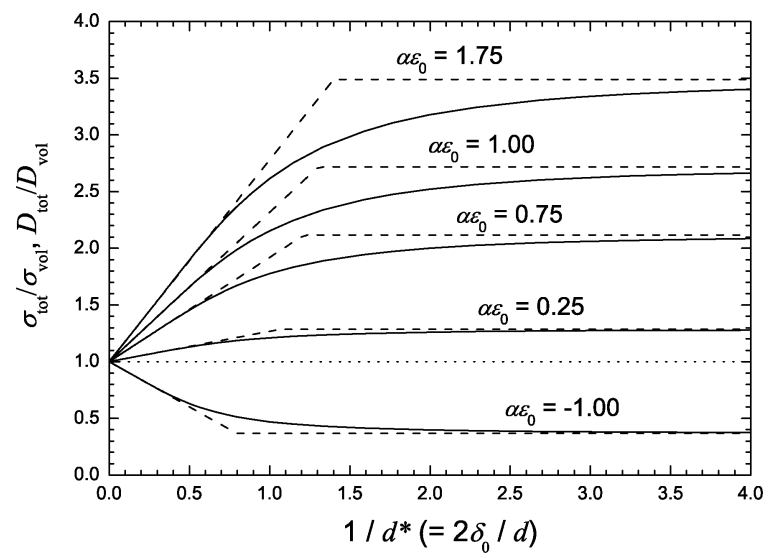

Fig. 4 Solid lines: relative ionic conductivity $\sigma_{\mathrm{tot}} / \sigma_{\mathrm{vol}}$ according to eqn (32) vs. the reciprocal film thickness parameter $1 / d^{*}\left(=2 \delta_{0} / d\right)$. Dashed lines: initial slopes and limiting interface conductivities. For the parameter $\alpha \varepsilon_{0}$ typical values found in ceramic thin films are used $(Y=200 \mathrm{GPa}, \nu=0.3$, $T=823 \mathrm{~K}, \Delta V^{\#}=2.0 \mathrm{~cm}^{3} \mathrm{~mol}^{-1}$ and $\left.\varepsilon_{0}=-1.8 \% \ldots .2 .2 \%\right)$. 


\subsection{Limit for very thick layers}

In the case of "thick" films any superposition of the interface strain fields is negligible. The integral in eqn (32) can be approximated, $\uparrow$ if $d \gg 2 \delta_{0}$, i.e. $d^{*} \gg 1$ :

$$
\frac{\sigma_{\mathrm{tot}}\left(d^{*} \gg 1\right)}{\sigma_{\mathrm{vol}}} \approx \frac{1}{d^{*}} \int_{0}^{d^{*}} \exp \left[\alpha \varepsilon_{0} \exp \left(z^{\prime}-d^{*}\right)\right] \mathrm{d} z^{\prime}
$$

The integral in eqn (34) of the type $\int \exp [a \exp (t)] \mathrm{d} t$ can be solved analytically:\|

$$
\frac{\sigma_{\mathrm{tot}}\left(d^{*} \gg 1\right)}{\sigma_{\mathrm{vol}}} \approx \frac{1}{d^{*}}\left[\operatorname{Ei}\left(\alpha \varepsilon_{0}\right)-\operatorname{Ei}\left(\alpha \varepsilon_{0} \mathrm{e}^{-d^{*}}\right)\right]
$$

For the limit $d^{*} \gg 1$ further simplifications can be made:**

$$
\frac{\sigma_{\mathrm{tot}}\left(d^{*} \gg 1\right)}{\sigma_{\mathrm{vol}}} \approx 1+\left[\operatorname{Ei}\left(\alpha \varepsilon_{0}\right)-\ln \left|\alpha \varepsilon_{0}\right|-\gamma\right] \frac{1}{d^{*}}
$$

Thus, eqn (32) can be approximated with a linear relation. The initial slope $\operatorname{Ei}\left(\alpha \varepsilon_{0}\right)-\ln \left|\alpha \varepsilon_{0}\right|-\gamma$ depends only on the parameter $\alpha$ and the mismatch induced elastic strain $\varepsilon_{0}$. For infinitely thick films $d^{*} \rightarrow \infty$, eqn (38) and thus eqn (32) will reach a limiting value of 1 , i.e. interfacial strain is negligible for geometrical reasons.

Ionic conductivity or diffusivity data, evaluated from experimental studies, as compiled in Table 1 , can only be plotted directly $v s$. the reciprocal film thickness $1 / d$, as $\delta_{0}$ is not known a priori:

$$
\frac{\sigma_{\mathrm{tot}}\left(d^{*} \gg 1\right)}{\sigma_{\mathrm{vol}}} \approx 1+2 \delta_{0}\left[\operatorname{Ei}\left(\alpha \varepsilon_{0}\right)-\ln \left|\alpha \varepsilon_{0}\right|-\gamma\right] \frac{1}{d}
$$

In a plot of $\sigma_{\text {tot }} / \sigma_{\mathrm{vol}}$ or $D_{\text {tot }} / D_{\mathrm{vol}} v s$. $1 / d$ the width of the strained interface region $\delta_{0}$ is part of the initial slope $2 \delta_{0}\left[\operatorname{Ei}\left(\alpha \varepsilon_{0}\right)-\ln \left|\alpha \varepsilon_{0}\right|-\gamma\right]$. Thus, the measured initial slope depends also on the diameter $l$ of the film crystallites, see eqn (22). In Fig. 5 the relative ionic conductivity $\sigma_{\text {tot }} / \sigma_{\mathrm{vol}}$ for $\alpha \varepsilon_{0}=1.0$ and a Poisson ratio $\nu=0.3$ is plotted for typical values of the crystallite diameter $l$, found in vacuum deposited thin films.

Clearly, not only the film thickness $d$ but also the crystallite morphology within the layers is an important parameter, influencing the strain effect on ionic transport. The shape of the crystallites, i.e. the ratio between height (identical with film

I If $d^{*} \gg 1$ one can approximate $\cosh d^{*} \approx 1 / 2 \mathrm{e}^{d^{*}}$. Since the integration variable $z^{\prime}$ varies only between 0 and $d^{*}$, the expression in the exponent of eqn (32) can be simplified:

$$
\frac{\cosh z^{\prime}}{\cosh d^{*}} \approx \mathrm{e}^{z^{\prime}-d^{*}}+\mathrm{e}^{-z^{\prime}-d^{*}} \approx \mathrm{e}^{z^{\prime}-d^{*}}
$$

$\|$ Using the exponential integral function $\operatorname{Ei}(x)=\int_{-\infty}^{x} \frac{\mathrm{e}^{t}}{t} \mathrm{~d} t$ one can derive:

$$
\int_{0}^{x} \mathrm{e}^{a \mathrm{e}^{t}} \mathrm{~d} t=\operatorname{Ei}\left(a \mathrm{e}^{x}\right)-\operatorname{Ei} a
$$

** A series expansion can be made for the exponential integral function. The infinite sum term can be neglected for small values of the functional argument:

$$
\begin{aligned}
& \operatorname{Ei}\left(\alpha \varepsilon_{0} \mathrm{e}^{-d^{*}}\right)= \gamma+\ln \left|\alpha \varepsilon_{0} \mathrm{e}^{-d^{*}}\right|+\sum_{n=1}^{\infty} \frac{\left(\alpha \varepsilon_{0} \mathrm{e}^{-d^{*}}\right)^{n}}{n \cdot n !} \\
& \approx \gamma+\ln \left|\alpha \varepsilon_{0}\right|-d^{*} \quad \text { if } \quad d^{*} \gg 0 \\
& \quad \text { with } \quad \gamma=0.577215665 \ldots \text { (Euler's constant) }
\end{aligned}
$$

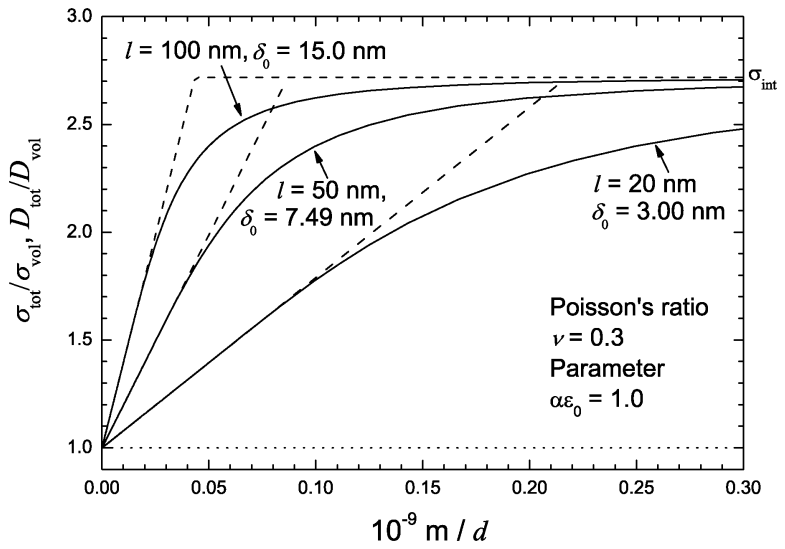

Fig. 5 Solid lines: relative ionic conductivity $\sigma_{\text {tot }} / \sigma_{\mathrm{vol}}$ according eqn (32) vs. the reciprocal film thickness $1 / d$ for typical values of the diameter $/$ of the crystallites in the thin film $\left(\alpha \varepsilon_{0}=1.0\right.$ and $\left.\nu=0.3\right)$. Dashed lines: initial slopes and limiting interface conductivity.

thickness) $d$ and diameter $l$ controls the volume fraction of the strained interface regions relative to the unstrained bulk, see eqn (22). For the same film thickness $d$, the strain effect on interface transport increases with increasing diameter $l$ of the crystallites. However, if the diameter of the crystallites decreases in the same proportion as the layer thickness, $l \sim d$, the effect cancels out and the overall transport coefficients $\sigma_{\text {tot }}$ and $D_{\text {tot }}$ remain constant $\left(2 \delta_{0} / d=\right.$ const. $)$.

\subsection{Limits for very thin layers}

In the case of very thin films the elastic strain fields, originating from both phase boundaries, overlap to a large extent. The integral in eqn (32) can be approximated, $\dagger \dagger$ if $d \ll 2 \delta_{0}$, i.e. $d^{*} \ll 1$ :

$$
\frac{\sigma_{\mathrm{tot}}\left(d^{*} \ll 1\right)}{\sigma_{\mathrm{vol}}} \approx \frac{1}{d^{*}} \int_{0}^{d^{*}} \exp \left(\alpha \varepsilon_{0}\right) \mathrm{d} z^{\prime}=\exp \left(\alpha \varepsilon_{0}\right)
$$

Thus, eqn (32) will reach a limiting value of $\mathrm{e}^{\alpha \varepsilon_{0}}$. As well as the initial slope for very thick layers, the limiting value of the relative ionic conductivity $\sigma_{\text {tot }} / \sigma_{\text {vol }}$ for very thin layers only depends on the parameter $\alpha$ and the mismatch induced elastic strain $\varepsilon_{0}$ (see Fig. 4).

Directly at the phase boundaries the maximum isotropic pressure and thus the maximum effect on the local ionic transport should be expected. Consequently, the limiting value $\sigma_{\text {tot }}\left(d^{*} \ll 1\right)$ in eqn $(41)$ can be defined as the (maximum) interface ionic conductivity $\sigma_{\text {int }}$ :

$$
\frac{\sigma_{\text {int }}}{\sigma_{\mathrm{vol}}}=\mathrm{e}^{\alpha \varepsilon_{0}}
$$

$\dagger \dagger$ If $\left|d^{*}\right| \ll 1$ one can approximate $\cosh d^{*} \approx 1$. Because, the integration variable $z^{\prime}$ varies only between 0 and $d^{*}$ the expression in the exponent of eqn (32) can be simplified to:

$$
\frac{\cosh z^{\prime}}{\cosh d^{*}} \approx \mathrm{e}^{z^{\prime}}+\mathrm{e}^{-z^{\prime}} \approx 1+0
$$


Table 2 Own experimental studies on (0001) $\mathrm{Al}_{2} \mathrm{O}_{3} / \mathrm{RE}_{2} \mathrm{O}_{3} /\left[\mathrm{YSZ} / \mathrm{RE}_{2} \mathrm{O}_{3}\right] \times n$ multilayers $(\mathrm{RE}=\mathrm{Lu}$, Sc and $\mathrm{Y}$ ). The mismatch is calculated for a commensurate relation of $2: 1$ of the unit cell parameter $\left(a_{Y S Z}: a_{R_{2}} \mathrm{O}_{3}\right)$. The width $\delta_{0}$ of the strained interface region is determined by non-linear curve fitting, using eqn (43), the value of the parameter $\alpha$ and the data plotted in Fig. 7. RSD is the standard deviation of $\delta_{0}$, calculated from the residue. The (average) diameters 1 of the film crystallites are calculated according to eqn (22), using the fit parameter $\delta_{0}$. The initial slopes are (a) calculated according to eqn (39) and (b) determined by linear regression of the onset of the plotted data

\begin{tabular}{|c|c|c|c|c|c|c|c|c|c|}
\hline Multilayer system & $f_{\mathrm{YSZ} / \mathrm{RE}_{2} \mathrm{O}_{3}} / \%$ & Experimental studies & Temperature $/{ }^{\circ} \mathrm{C}$ & \multicolumn{3}{|c|}{ Fit using eqn (43) } & \multicolumn{3}{|c|}{ Initial slope/nm } \\
\hline $\mathrm{YSZ} / \mathrm{Sc}_{2} \mathrm{O}_{3}$ & -4.37 & $\begin{array}{l}\text { Conductivity } \\
\text { Diffusion }^{13}\end{array}$ & $\begin{array}{l}560 \\
550\end{array}$ & $\begin{array}{l}53.4 \\
54.0\end{array}$ & $\begin{array}{l}4.4 \\
6.3\end{array}$ & $\begin{array}{l}0.1 \\
0.1\end{array}$ & $\begin{array}{l}32 \\
46\end{array}$ & $\begin{array}{l}-7.9 \\
-11.4\end{array}$ & $\begin{array}{l}-6.1 \pm 0.8 \\
-8.5 \pm 0.2\end{array}$ \\
\hline $\mathrm{YSZ} / \mathrm{Lu}_{2} \mathrm{O}_{3}$ & 1.02 & $\begin{array}{l}\text { Conductivity }{ }^{24,60} \\
\text { Diffusion }\end{array}$ & $\begin{array}{l}560 \\
\text { Not measured }\end{array}$ & 53.4 & $(-0.3)$ & 0.3 & $(-2.1)$ & $(-0.17)$ & $-1.4 \pm 2.4$ \\
\hline
\end{tabular}

\subsection{An approximation function for the relative ionic conductivity}

There is no analytical solution for the parametric integral in eqn (32). An approximation function can be given, by combining the limiting values for very thin and very thick layers: $\$$

$$
\begin{aligned}
\frac{\sigma_{\mathrm{tot}}\left(d^{*}\right)}{\sigma_{\mathrm{vol}}} \approx & \frac{1}{d^{*}}\left(\mathrm{Ei} \frac{\alpha \varepsilon_{0}}{1+\mathrm{e}^{-2 d^{*}}}-\mathrm{Ei} \frac{\alpha \varepsilon_{0}}{1+\mathrm{e}^{2 d^{*}}}\right) \\
& +\exp \frac{\alpha \varepsilon_{0}}{2 \cosh d^{*}}\left(\exp \frac{\alpha \varepsilon_{0}}{2 \cosh d^{*}}-2\right)
\end{aligned}
$$

The function in eqn (44) is a good approximation for lattice mismatches below $10 \%$, using values of the material constants typically found in ceramic ionic conductors. The highest derivations are found in the intermediate range for $d^{*} \approx 1$.

\section{Discussion}

In the following we will discuss and compare the experimental studies from the literature ${ }^{5,11,49-51}$ and own studies ${ }^{6,9,12,13,24,60}$ performed on extrinsic oxide ion conductors from category (i), as summarised in Table 1, with the improved strain model. In these studies the total ionic conductivity $\sigma_{\text {tot }}$ and diffusion coefficient $D_{\text {tot }}$ for oxide ion transport in multilayers consisting of the extrinsic conductor YSZ are measured parallel to the phase boundaries, as described by the strain model (see Fig. 3). For a proof of the introduced theoretical model on a quantitative-and not only on a qualitative level-the basic assumption limiting its applicability has to be considered. Only studies using systems with plain, parallel orientated and coherent films can be quantitatively discussed.

\# An approximation for the parametric integral $\int_{0}^{x} \mathrm{e}^{a \cosh t} \mathrm{~d} t$ can be found when considering that the hyperbolic cosine converges to $1 / 2 \mathrm{e}^{ \pm t}$ for $t \rightarrow \pm \infty$ and to 1 for $t \rightarrow 0$. The slope at $x \rightarrow 0$, i.e. $\mathrm{d} / \mathrm{d} x \int_{0}^{x} \mathrm{e}^{a \cosh t} \mathrm{~d} t$ should be $\mathrm{e}^{a}$. Thus, for $x \rightarrow \pm \infty$ it can be approximated by the exponential integral function and for $x \rightarrow 0$ by a linear function:

$$
\int_{0}^{x} \mathrm{e}^{a \cosh t} \mathrm{~d} t \approx \operatorname{Ei}\left(\frac{a}{2} \mathrm{e}^{x}\right)-\operatorname{Ei}\left(\frac{a}{2} \mathrm{e}^{-x}\right)+\mathrm{e}^{\frac{a}{2}}\left(\mathrm{e}^{\frac{a}{2}}-2\right) x
$$

The maximum deviation of this approximation function from the exact solution is about $5 \%$.
In the studies from the literature and own studies, summarised in Tables 1 and 2, the ionic transport in the multilayer and thin film samples is always measured parallel to phase boundaries. The total $\mathrm{O}^{2-}$-conductivity is determined by impedance spectroscopy and the $\mathrm{O}^{2-}$-diffusion coefficients by ${ }^{18} \mathrm{O}$-tracer experiments. For an extrinsic ionic conductor like YSZ or CGO, the relative change of the ionic conductivity $\sigma_{\mathrm{tot}} / \sigma_{\mathrm{vol}}$ (to the bulk value) is identical to the relative change of the diffusion coefficient of the mobile ions $D_{\text {tot }} / D_{\text {vol }}$, if there is no additional electronic conduction: $\S$

$$
\frac{\sigma_{\mathrm{tot}}}{\sigma_{\mathrm{vol}}}=\frac{D_{\mathrm{tot}}}{D_{\mathrm{vol}}} \frac{c_{\mathrm{tot}}}{c_{\mathrm{vol}}}
$$

In an extrinsic ionic conductor, the concentration of mobile charge is virtually constant due to the high doping concentration. Thus, the concentration of mobile $\mathrm{O}^{2-}$-ions close to the phase boundaries should be essentially the same as in the bulk phase, $c_{\mathrm{tot}} \approx c_{\mathrm{vol}}$. For this reason, the relative changes in the transport parameters $\sigma_{\mathrm{tot}} / \sigma_{\mathrm{vol}}$ and $D_{\mathrm{tot}} / D_{\mathrm{vol}}$ for $\mathrm{O}^{2-} v s$. the reciprocal film thickness $1 / d$ are comparable and can be combined and plotted in the same diagram (see Fig. 7).

\subsection{Material parameters of YSZ}

In the case of a pure strain based effect on the interface transport, the magnitude of the expected increase (or decrease) of the total ionic conductivity and diffusivity and thus the slope in a $\sigma_{\text {tot }} / \sigma_{\text {vol }}$ or $D_{\text {tot }} / D_{\text {vol }}$ vs. $1 / d$ plot can be estimated, using eqn (39). The value of the dimensionless parameter $\alpha$ can be calculated solely from literature data for the elastic properties and activation volume:

Activation volume of YSZ. Park et al. have measured a value of $2.08 \mathrm{~cm}^{3} \mathrm{~mol}^{-1}$ for the activation volume $\Delta V^{\#}$ of YSZ $\left(9.5 \mathrm{~mol} \% \mathrm{Y}_{2} \mathrm{O}_{3}\right){ }^{73}$ The experimental study was performed at $750{ }^{\circ} \mathrm{C}$, applying uniaxial pressure. This is in accordance with other studies on (extrinsic) fluorite type anionic conductors, ${ }^{74-81}$ providing typically values of $2-4 \mathrm{~cm}^{3} \mathrm{~mol}^{-1}$. The activation volume $\Delta V^{\#}$ is strongly dependent on the lattice structure. Rock salt type ionic conductors have generally higher values in the range of $6-10 \mathrm{~cm}^{3} \mathrm{~mol}^{-1} \cdot{ }^{72,82}$

$\S \S$ Considering the Nernst-Planck relation: $\sigma_{i} /\left(z_{i} F\right)^{2}=D_{i} c_{i} / R T$. 
Elastic properties of YSZ. The model is derived for a material with isotropic elastic properties. The elastic parameters for cubic YSZ, Young's modulus $Y$ and the Poisson ratio $\nu$, change considerably with the crystallographic direction. ${ }^{83-85}$ Moreover, Young's modulus is also temperature dependent. ${ }^{84-90}$ The isotropic (average) Young's modulus of YSZ doped with $10 \mathrm{~mol} \%$ $\mathrm{Y}_{2} \mathrm{O}_{3}$ decreases considerably from $210 \mathrm{GPa}$ to $167 \mathrm{GPa}$ in the temperature range between 25 and $400{ }^{\circ} \mathrm{C}$. The Poisson ratio does only change slightly from 0.36 to $0.37:^{89}$

\begin{tabular}{lllllll}
\hline Temperature $/{ }^{\circ} \mathrm{C}$ & 25 & 400 & 450 & 500 & 550 & 600 \\
\hline Young's modulus/GPa & 210 & 167 & 167 & 168 & 168 & 169 \\
Poisson ratio & 0.36 & 0.37 & 0.37 & 0.37 & 0.37 & 0.36
\end{tabular}

At room temperature in the doping range from 5 to $15 \mathrm{~mol} \%$ $\mathrm{Y}_{2} \mathrm{O}_{3}$ there is no pronounced composition dependence of the elastic parameters. ${ }^{85,91}$ At higher temperatures also a considerably composition dependence can be noticed. Thus, for temperatures between 400 and $600{ }^{\circ} \mathrm{C}$ we calculate values between 65.7 and 50.4 for the parameter $\alpha$.

\subsection{Experimental studies on YSZ multilayers from the literature}

The maximum misfit observed in experimental studies on polycrystalline thin films, respectively, multilayers with columnar crystallite structure and (quasi-)coherent phase boundaries may not exceed 6 to $10 \%$. The average crystallite sizes of films prepared by gas phase deposition are typically in the range of 20 to $100 \mathrm{~nm}$. The highest misfits will only be reached in thin films, consisting of (columnar) crystallites with a very small diameter. According to eqn (42), this results in an increase of the interface transport coefficients compared to the bulk value of about 10 at maximum, but in the case of such small crystallites the effect of blocking grain boundaries will also be maximised. The maximum slope as defined in eqn (39) may not exceed values of $s_{\sigma}=30 \mathrm{~nm}$.

Unfortunately, a detailed and quantitative discussion of all experimental studies from the literature in category (i) is not possible. In the case of the studies of Karthikeyan et al. on multilayer samples, consisting of random orientated YSZ films and amorphous insulating layers, it is difficult to estimate the actual interface strain. ${ }^{49}$ In the studies of Pergolesi and Li et al. multilayers consisting of two ionic conductors with comparable $\mathrm{O}^{2-}$-conductivity were used. ${ }^{11,51}$ If a misfit is relaxed by tensile strain in the first ion conducting phase and by compressive strain in the second phase, as assumed in our model, the increased transport in the first phase will be (partially) canceled out or overcompensated by the decreased transport in the second phase (or vice versa). The resulting effect on the total conductivity or the diffusion coefficient may depend on the ratio of the elastic properties, the activation volumes as well of the layer thicknesses. In spite of the problem that no definite estimation can be made, all measured slopes of the studies from category (i) are in the expected range below $s_{\sigma}=30 \mathrm{~nm}$.
In the case of the experimental study of Pergolesi et al. the phase boundaries of the used $\mathrm{YSZ} / \mathrm{CeO}_{2}$-multilayer samples were also heavily rugged. ${ }^{51,92}$ Mismatch induced coherency strain can be reduced by introduction of misfit dislocations but also by tilting of the interface planes. This may be the cause why any strain effect is suppressed (despite 5.2\% misfit) and why the authors observe no conductivity effect.

There is only one exceptional study with an extraordinary large effect with a slope in the order of $10^{8} \mathrm{~nm}$. As discussed in the beginning this is probably a misinterpretation of the effects caused by electronic conduction or experimental issues. ${ }^{61-63}$

\subsection{Own experimental studies on the YSZ/rare earth metal oxide multilayer}

Preparation of model systems. Due to the lack of experimental studies which allow a quantitative evaluation of the strain state in the interface, we have performed own studies on multilayers consisting of cubic fluorite-type YSZ and ionic transport blocking cubic bixbyite-type rare earth metal sesquioxides..$^{6,9,12,13,24,60}$ The bixbyite structure $(I a \overline{3})$ is closely related to the fluorite structure $(F m \overline{3} m)$. Essentially, the bixbyite unit cell has twice the lattice parameter of the fluorite unit cell. Only $2 / 3$ of the original fluorite anion lattice sites are occupied. In our recent studies multilayer samples with different rare earth metal sesquioxides $\mathrm{RE}_{2} \mathrm{O}_{3}$ with $\mathrm{RE}=\mathrm{Lu}, \mathrm{Sc}$ and $\mathrm{Y}$ were prepared by pulsed laser deposition on (0001) $\mathrm{Al}_{2} \mathrm{O}_{3}$ substrates. YSZ with a doping level of $9.5 \mathrm{~mol} \% \mathrm{Y}_{2} \mathrm{O}_{3}$ was used. The lattice parameter of the used bixbyite-type rare earth metal sesquioxide $\mathrm{RE}_{2} \mathrm{O}_{3}$ and the fluorite-type YSZ have approximately a 2:1 ratio. An overview of the experimental studies is given in Table 2 .

The samples were prepared by pulsed laser deposition (PLD) and characterised by SEM, TEM/SAED and XRD/pole figure measurements. Despite the considerable mismatch between $\mathrm{YSZ}, \mathrm{Y}_{2} \mathrm{O}_{3}$, and $\mathrm{Sc}_{2} \mathrm{O}_{3}$, in the phase boundaries of these multilayers, respectively, only very few mismatch dislocations are formed. Dislocations are located only in and close to the grain boundaries. The phase boundaries in these studies are flat on an atomic level and can be treated as (quasi-)coherent with an ideal 1:1 match of the lattice plains. By changing the rare earth metal sesquioxide, i.e. the lattice parameter, the mutual mismatch $f_{\mathrm{ZrO}_{2} / \mathrm{RE}_{2} \mathrm{O}_{3}}$ can be systematically varied. Thus, the chosen combination of cubic oxides can be used as (nearly) ideal model systems to study strain based effects.

As seen in Fig. 6, the crystallites in all prepared multilayers have only constrained diameters below $100 \mathrm{~nm}$. Thus, the interface strain can be considerably relaxed by local deformation. The average crystallite sizes of all samples are in the same range (see below). Using (0001) $\mathrm{Al}_{2} \mathrm{O}_{3}$ substrates, the crystallites in the thin films of the $\mathrm{YSZ} / \mathrm{Y}_{2} \mathrm{O}_{3}$ - and $\mathrm{YSZ} / \mathrm{Sc}_{2} \mathrm{O}_{3}$-multilayer have only one preferential orientation, (111) YSZ/(111) $\mathrm{RE}_{2} \mathrm{O}_{3}$ $(\mathrm{RE}=\mathrm{Y}, \mathrm{Sc})$. In the case of a YSZ/ $\mathrm{Lu}_{2} \mathrm{O}_{3}$-multilayer also other orientations like (110) YSZ/(110) $\mathrm{Lu}_{2} \mathrm{O}_{3}$ and (100) YSZ/(100) $\mathrm{Lu}_{2} \mathrm{O}_{3}$ were found. Details of the experimental setup and the characterisation are given in the cited studies.

\subsection{Fitting of the data of own experimental studies}

The relative conductivity and diffusion data in Fig. 7 can be fitted with the approximation function in eqn (43) to validate 

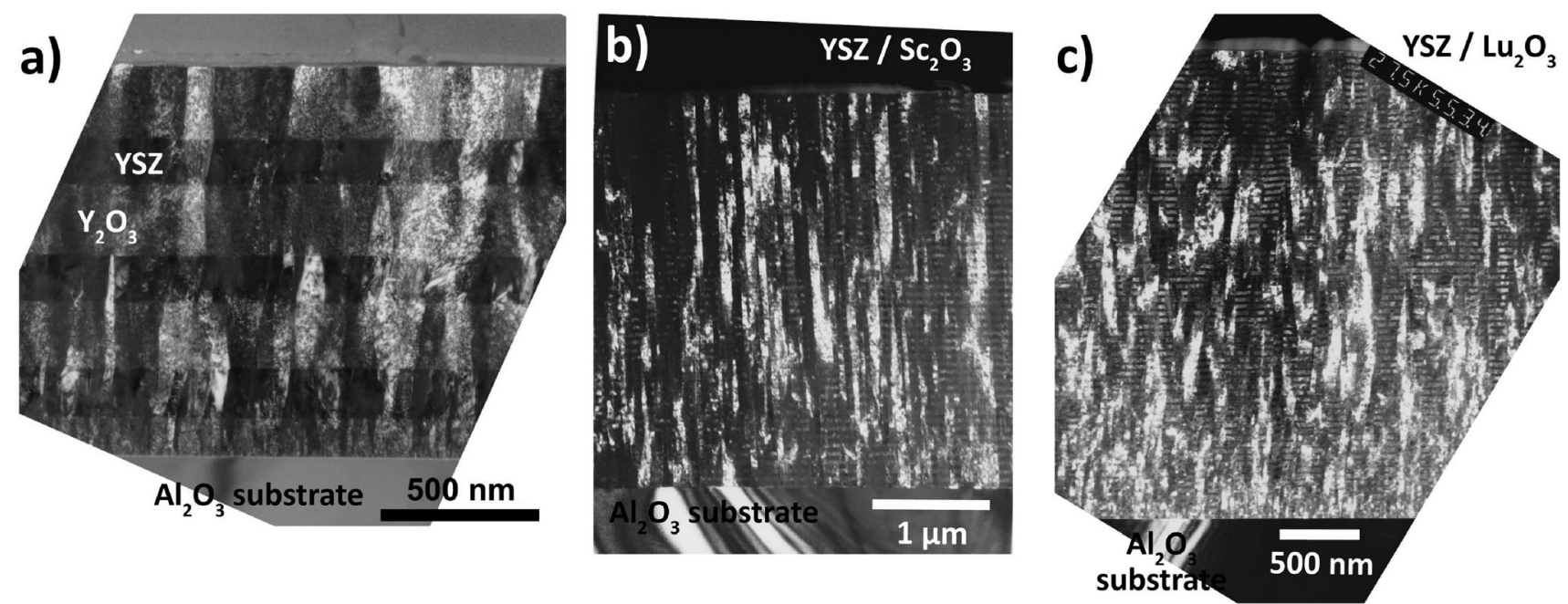

Fig. 6 Dark field (DF) TEM micrographs of $\mathrm{Al}_{2} \mathrm{O}_{3} / \mathrm{RE}_{2} \mathrm{O}_{3} /\left(\mathrm{YSZ} / \mathrm{RE}_{2} \mathrm{O}_{3}\right) \times n$ multilayers. (a) $\mathrm{YSZ} / \mathrm{Y}_{2} \mathrm{O}_{3}$ with $n=3$, (b) $\mathrm{YSZ} / \mathrm{SC}_{2} \mathrm{O}_{3}$ with $n=50$ and $\mathrm{YSZ} / \mathrm{Lu}_{2} \mathrm{O}_{3}$ with $n=70$ YSZ layers. ${ }^{6,9,60}$ All multilayers show a columnar microstructure. The (average) diameter of the crystallites is below $100 \mathrm{~nm}$.

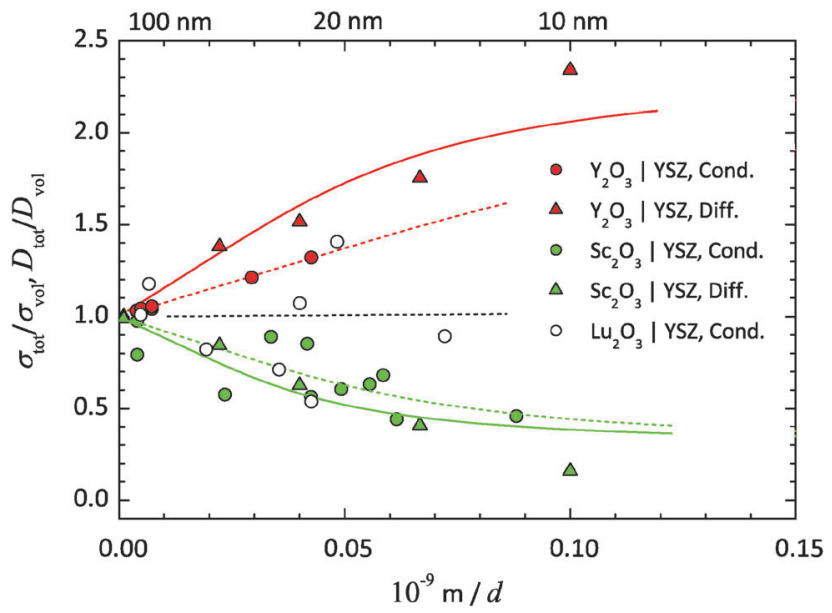

Fig. 7 Relative $\mathrm{O}^{2-}$-conductivity and diffusion coefficients, $\sigma_{\text {tot }} / \sigma_{\mathrm{vol}}$ and $D_{\text {tot }} / D_{\text {vol }}$, from the YSZ/RE $\mathrm{O}_{3}$-multilayer studies (RE $=\mathrm{Y}, \mathrm{Lu}, \mathrm{Sc}$ ) listed in Table 2 vs. the reciprocal thickness $1 / d$ of the YSZ layers. The blue and red curves are fitted to the data, using eqn (32) and the values for the parameter $\alpha$ in Table 2 .

the introduced model. The material dependent parameter $\alpha$ of YSZ for the experimental temperatures is calculated from literature data ${ }^{89}$ and also listed in Table 2 . The only fitting parameter is the width $\delta_{0}$ of the strained interface region.

YSZ $/ \mathbf{Y}_{2} \mathbf{O}_{3}$. The lattice parameters of YSZ $\left(9.5 \mathrm{~mol} \% \mathrm{Y}_{2} \mathrm{O}_{3}\right)$ amount to $5.143 \AA$ and of $\mathrm{Y}_{2} \mathrm{O}_{3}$ to $10.604 \AA{ }^{93-98}$ Due to the positive lattice mismatch of $+3.04 \%$, a tensile strain $\varepsilon_{0}$ of $+1.52 \%$ relative to the YSZ layers should exist. The relative ionic conductivity and the diffusion coefficient, $\sigma_{\mathrm{tot}} / \sigma_{\mathrm{vol}}$ and $D_{\mathrm{tot}} / D_{\mathrm{vol}}$, are increasing with decreasing layer thickness $d$ (increasing density of interfaces). Thus, the initial slopes are positive. As expected from eqn (28), tensile strain results in an increased ionic transport coefficient in the interface region compared to the bulk. An increasing effect of tensile strain on the interface conductivity in extrinsic $\mathrm{O}^{2-}$-conductor is also clearly demonstrated by Li et al. and Sanna et al. for YSZ/GZO and YSZ/SDC multilayers. ${ }^{11,50}$ By fitting eqn (43) $\delta_{0}$ yields as $3.7 \mathrm{~nm}$ for the conductivity data and as $7.1 \mathrm{~nm}$ for the diffusion data. Using eqn (22) the (average) diameter $l$ of the film crystallites can be calculated from $\delta_{0}$. For the conductivity data one gets an (average) diameter of $27 \mathrm{~nm}$ and for the diffusion data of $51 \mathrm{~nm}$.

YSZ/Sc $\mathbf{S c}_{2} \mathbf{O}_{3}$. The lattice parameter of $\mathrm{Sc}_{2} \mathrm{O}_{3}$ equals $9.846 \AA{ }^{99}$ Due to the negative lattice mismatch of $-4.37 \%$ there is a compressive strain $\varepsilon_{0}$ of $-2.19 \%$ relative to the YSZ layers. As expected for compressive strain in the interfaces a decrease of the ionic conductivity and the diffusion coefficient has been measured. The initial slopes are negative. A decreasing effect of compressive interface strain on conductivity is also shown by Shen et al. for the YDC/CZO multilayer. ${ }^{15}$ By fitting, $\delta_{0}$ yields $4.4 \mathrm{~nm}$ for the conductivity data and $6.3 \mathrm{~nm}$ for the diffusion data. The (average) diameter $l$ of the film crystallites results $32 \mathrm{~nm}$ for the conductivity data and $46 \mathrm{~nm}$ for the diffusion data.

$\mathbf{Y S Z} / \mathbf{L u}_{2} \mathbf{O}_{3}$. The lattice parameters of $\mathrm{Lu}_{2} \mathrm{O}_{3}$ equal $10.391 \AA^{100,101}$ The small positive lattice mismatch of $+1.02 \%$ and thus the only small tensile strain $\varepsilon_{0}$ of $+0.52 \%$ should result to the least effect on phase boundary transport. Indeed, no clear trend can be seen, due to the strong scattering of the present conductivity data. A simple linear regression of the data yields a negative slope. By fitting eqn (43), $\delta_{0}$ will converge to a small but negative value, because of the specified positive strain. Due to the lack of accuracy of the experimental data, the obtained values for $\mathrm{YSZ} / \mathrm{Lu}_{2} \mathrm{O}_{3}$ (marked with round brackets) have no physical meaning. At the moment there are no diffusion data available.

Diameter of film crystallites from TEM. The average size of the columnar film crystallites of the multilayer samples can also be estimated from (HR)TEM investigations. This yields values for the film crystallite diameter $l$ in the order of 25-75 nm for the $\mathrm{YSZ} / \mathrm{Y}_{2} \mathrm{O}_{3}$-multilayer, respectively, in the order of 20-50 nm for the $\mathrm{YSZ} / \mathrm{Sc}_{2} \mathrm{O}_{3}$-multilayer, see Fig. 6(a) and (b). ${ }^{6,9}$ 
The smaller grain size is measured close to the $\mathrm{Al}_{2} \mathrm{O}_{3}$ substrate, the larger in the middle of the multilayer. Thus, the values for $l$ determined by fitting the conductivity data of about $30 \mathrm{~nm}$ and by fitting the diffusion data of about $50 \mathrm{~nm}$ are close to the values from the microscopic investigations.

In the case of the $\mathrm{YSZ} / \mathrm{Lu}_{2} \mathrm{O}_{3}$-multilayers the diameter $l$ cannot be determined by fitting because of the strong scattering of the present conductivity data. From (HR)TEM investigations values between 20 and $70 \mathrm{~nm}$ were determined for the (average) diameter $l$ of the YSZ crystallites, ${ }^{24,60}$ which are comparable to the values obtained for $\mathrm{YSZ} / \mathrm{Y}_{2} \mathrm{O}_{3}$ - and $\mathrm{YSZ} / \mathrm{Sc}_{2} \mathrm{O}_{3}$-multilayers, see Fig. 6(c).

\subsection{Partial electronic conduction}

In the case of stoichiometric composition, bulk phase rare earth sesquioxides $\mathrm{Y}_{2} \mathrm{O}_{3}, \mathrm{Sc}_{2} \mathrm{O}_{3}$ and $\mathrm{Lu}_{2} \mathrm{O}_{3}$ are insulators for ionic and electronic transport ${ }^{6,9,24,60}$ and bulk phase YSZ is virtually a pure ionic conductor with a negligible electronic transfer number. For thin film or multilayer samples, additional electronic conductivity due to a partial reduction of the bulk phases or a change of the electronic band structure in the interface region is discussed. ${ }^{63,102}$ Also experimental artefacts originating from external short-circuit paths are often considered, but can be safely excluded in the case of our multilayers-as their resistance is always much lower than the resistance of the substrate and sample holder. ${ }^{61,62}$

The absence of electronic partial conductivity can be verified by comparing the results of the ionic conductivity with ${ }^{18} \mathrm{O}$-tracer diffusion measurements. ${ }^{12,13}$ In a diffusion experiment only ionic transport is measured. In a conductance experiment a possible electronic partial conductivity may be included in the total conductivity. In all of our studies $\sigma_{\text {tot }} / \sigma_{\mathrm{vol}}$ is equal or at least smaller than $D_{\text {tot }} / D_{\text {vol }}$. One can conclude that neither a significant electronic partial conduction nor leakage currents through the substrate or the ceramic sample holder play a measurable role. This holds as long as there is no strong variation of the (average) crystallite diameter and no depletion of mobile oxygen vacancies in the interface region, which might overcompensate an increase of the total conductivity caused by additional electronic conduction.

\subsection{Grain boundaries}

The grain boundaries between the crystallites in ionic conducting layers may influence the total ionic conductivity of the multilayer. Depending on the mutual orientation of adjacent crystallites, i.e. on the grain boundary structure (from low angle to high angle grain boundaries), ionic transport across (perpendicular) the grain boundaries is often hampered compared to the bulk. ${ }^{103}$

In addition to the blocking effect at grain boundaries also additional non-equilibrium lattice defects, like local disordered regions or dislocation loops in the volume of the crystallites, can reduce the measured total ionic transport. Such lattice defects can locally influence the point defect equilibria and alter the migration enthalpy. ${ }^{104,105}$ In the case of thin films, non-equilibrium lattice defects in particular originate from the vapour deposition techniques used for preparation. Such defects can only relax if sufficient diffusional (cationic and anionic) transport through the volume is possible. This can be safely achieved at a preparation temperature or a subsequent heat treatment above $1 / 2$ to $3 / 4$ of the melting point $T_{\text {melt }}{ }^{106,107}$ All multilayers were subjected to a heat treatment at 800 before performing the measurements. This is only $1 / 3$ of the melting temperature of YSZ $\left(\sim 2700{ }^{\circ} \mathrm{C}\right)$.

The blocking effect of grain boundaries and additional nonequilibrium lattice defects within the crystallites in micro-/ nanocrystalline films can reduce the mean ionic transport coefficients-compared to (bulk) single crystal samples-by up to one order of magnitude. Depending on the grain orientation and the degree of the film texture even anisotropic conductivity can be expected. By and large, conductivity effects caused by the texture, grain boundary structure and nonequilibrium lattice defects may be in the same order of magnitude as strain-based effects at hetero-phase boundaries. We emphasise that our model in Section 3 describes only the strain effect caused by hetero-phase boundaries. Any other blocking effect has to be treated separately. This is perfectly reasonable, as the (absolute) conductivity of the bulk film does not represent a model parameter-we only scale the strain effect by comparison with the film bulk conductivity.

In essence, a prerequisite for the reproducible investigation of strain effects are multilayer samples with a comparable texture, grain boundary density and lattice defect density in the crystallites. The ionic conductivity for unstrained regions of the films is in fact reduced compared to a single crystal, but should be roughly sample independent. This can be only achieved if all samples are prepared with the same deposition technique, using well reproducible conditions. As described before, in the case of (own) experimental studies on $\mathrm{YSZ} / \mathrm{RE}_{2} \mathrm{O}_{3}$ multilayers this could be assured by using always the same deposition parameters for PLD preparation, the same heat treatment before performing the ionic transport measurements, and by subsequent XRD and TEM analysis. $9,12,13,48$

By analysing the results from the improved strain based model, one finds that the (average) diameter $l$ of the crystallites in the ion conducting layers on its own is a crucial parameter in addition to the layer thickness $d$, determining the extent of the strain effect on ionic transport. The (average) diameter of the crystallites directly influences the strain relaxation in the interface regions, i.e. the width of the strained region $\delta_{0}$ and thus the measured initial slopes, see eqn (22). By combining eqn (39) with eqn (22) one finds:

$$
\frac{\sigma_{\mathrm{tot}}\left(d^{*} \gg 1\right)}{\sigma_{\mathrm{vol}}} \approx 1+\left[\operatorname{Ei}\left(\alpha \varepsilon_{0}\right)-\ln \left|\alpha \varepsilon_{0}\right|-\gamma\right] \sqrt{\frac{1}{6} \frac{1-\nu}{1+\nu}} \frac{l}{d}
$$

In multilayer samples with a decreasing (average) diameter $l$ of the film crystallites as a function of the layer thickness $d$, i.e. with a linear dependence like $l \sim d$, the geometric dependence on the measured total transport properties disappears or will be diminished.

\subsection{Point defect association equilibria}

In heavily doped extrinsic ionic conductors like YSZ or CGO the mobile oxygen vacancies can form associates with the 
immobile dopant cations, which are charged relative to the regular cations. In the case of YSZ, the formation of $\left\{\mathrm{V}_{\mathrm{O}}^{\bullet \bullet}, \mathrm{Y}_{\mathrm{Zr}}^{\prime}\right\}$ pairs has then to be considered, which depends on the $\mathrm{Y}_{2} \mathrm{O}_{3}$ doping level:

$$
\begin{gathered}
\mathrm{Y}_{2} \mathrm{O}_{3}=2 \mathrm{Y}_{\mathrm{Zr}}^{\prime}+3 \mathrm{O}_{\mathrm{O}}^{\times}+\mathrm{V}_{\mathrm{O}}^{\bullet \bullet} \\
\mathrm{V}_{\mathrm{O}}^{\bullet \bullet}+\mathrm{Y}_{\mathrm{Zr}}^{\prime}=\left\{\mathrm{V}_{\mathrm{O}}^{\bullet \bullet}, \mathrm{Y}_{\mathrm{Zr}}^{\prime}\right\}
\end{gathered}
$$

In the presented model in Section 3 the mismatch induced strain is linked in a kinetic first-order approach with the ionic conductivity only by the pressure dependence of the free activation enthalpy $\Delta G^{\#}$ for ion jumps, see eqn (26), which governs the rate of successful jumps. The concentration of mobile point defects is assumed to be constant. When taking point defect equilibria into account, as eqn (47), pressure, respectively, pressure gradients, can influence the concentration of mobile oxygen vacancies $c_{\mathrm{V}_{\mathrm{O}}}$. The defect association equilibrium in eqn (47) can be described by a mass action law with an equilibrium constant $K_{\text {ass }}$ and a free reaction enthalpy $\Delta G_{\text {ass }}^{0}$ :

$$
\frac{c_{\left\{\mathrm{v}_{\mathrm{O}}^{*}, \mathrm{Y}_{\mathrm{Zr}}^{\prime}\right\}}}{c_{\mathrm{V}_{\mathrm{O}}^{\bullet \bullet}} c_{\mathrm{Y}_{\mathrm{Zr}}^{\prime}}}=K_{\mathrm{ass}}=\mathrm{e}^{-\frac{\Delta G_{\mathrm{ass}}^{0}}{R T}}
$$

In the case of strong association, i.e. $c_{\mathrm{V}_{\mathrm{O}}} \ll c_{\mathrm{Y}_{\mathrm{Zr}}^{\prime}}, c_{\left\{\mathrm{V}_{\mathrm{O}}^{\bullet \bullet}, \mathrm{Y}_{\mathrm{Zr}}^{\prime}\right\}}$ and $c_{\mathrm{Y}_{\mathrm{Zr}}^{\prime}} \approx c_{\left\{\mathrm{V}_{\mathrm{O}}^{\bullet}, \mathrm{Y}_{\mathrm{Zr}}^{\prime}\right\}}$, eqn (48) can be approximated to:

$$
c_{\mathrm{V}_{\mathrm{O}}^{\bullet \bullet}} \approx 1 / K_{\mathrm{ass}}
$$

By inserting eqn (49) in (26) the pressure dependence of the ionic conductivity and the diffusion coefficient can be easily calculated. The influence of strain on the conductivity $\sigma$ is now controlled by sum of the activation volume $\Delta V^{\#}$ for the ionic motion and the reaction volume $\Delta V_{\text {ass }}^{0}$ of point defect association:

$$
\left(\frac{\partial \ln \sigma}{\partial p}\right)_{T}=-\frac{\Delta V^{\#}+\Delta V_{\mathrm{ass}}^{0}}{R T}
$$

The result for the diffusion coefficient $D$ is analogue. There are only very few experimental studies available providing data for the reaction volume of point defect association. ${ }^{108-111}$ Positive as well as negative values can be found. A reaction volume $\Delta V_{\text {ass }}^{0}$ in the order of $-9 \mathrm{~cm}^{3} \mathrm{~mol}^{-1}$ is estimated for the formation of $\left\{\mathrm{V}_{\mathrm{O}}^{\bullet \bullet}, \mathrm{Gd}_{\mathrm{Ce}}^{\prime}\right\}$ ion pairs in CGO. ${ }^{109,110}$ Thus, association effects may either enhance or diminish the effect of strain on ionic jumps.

In the case of weak association, i.e. $c_{\mathrm{V}_{\mathrm{O}}^{*}}, c_{\mathrm{Y}_{\mathrm{Zr}}^{\prime}} \gg c_{\left\{\mathrm{v}_{\mathrm{O}}^{*}, \mathrm{Y}_{\mathrm{Zr}}^{\prime}\right\}}$ and $c_{\mathrm{Y}_{\mathrm{Zr}}^{\prime}} / 2 \approx c_{\mathrm{V}_{\mathrm{O}}^{*}}$, eqn (48) can be approximated to:

$$
c_{\mathrm{V}_{\mathrm{O}}} \approx \frac{1}{2\left(K_{\mathrm{ass}}+1 / c_{\mathrm{Y}_{\mathrm{Zr}}^{\prime}}^{0}\right)}
$$

Hereby, $c_{\mathrm{Y}_{\mathrm{Zr}}^{\prime}}^{0}$ is the total concentration of $\mathrm{Y}^{3-}$ dopant cations. If $K_{\text {ass }} \ll 1 / c_{\mathrm{Y}_{\mathrm{Zr}}^{\prime}}^{0}$, point defect association can be neglected and the pressure dependence of the concentration of mobile oxygen vacancies $c_{\mathrm{V}_{\mathrm{O}}}$ will tend to zero. If the value of $K_{\text {ass }}$ has the same magnitude as $1 / c_{\mathrm{Y}_{\mathrm{Zr}}^{\prime}}^{0}$, the pressure dependence in the case of weak association is smaller compared to strong association. We assume that association effects can be neglected in this case, anyhow.

Clearly, mere transport experiments do not allow us to separate the effects of strain on defect mobility and on the concentration of mobile defects. But one should keep in mind that the effect on the concentration of mobile vacancies can be significant, once association is severe. The values of $\Delta V^{\#}$ and $\Delta V_{\text {ass }}^{0}$ can be of comparable magnitude and can either add up or cancel out.

We also like to add that the segregation of dopants or impurities in the vicinity of grain and phase boundaries may have an effect. In heavily doped extrinsic ionic conductors like YSZ or CGO the doping components $\mathrm{Y}_{2} \mathrm{O}_{3}, \mathrm{Gd}_{2} \mathrm{O}_{3}$, respectively, are often accumulated in the interfaces, depending on the preparation conditions. ${ }^{112,113}$ As state above, we could not find evidence for significant segregation at hetero-phase boundaries prepared by PLD, and therefore, we exclude it from further discussion. However, the possibility should be kept in mind, and under prolonged high temperature treatment segregation may occur even driven by local strain.

\section{Conclusions/summary}

\subsection{Phenomenological model and model experiments}

We introduce a theoretical model for the description of the ionic transport in strained polycrystalline ceramic thin films and multilayers, consisting of a solid electrolyte and an insulator. It is based on interface strain fields, originating from phase boundaries with a structural mismatch. Assuming exponentially decaying strain fields, an analytical expression is obtained for the ionic conductivity/ionic diffusion coefficient relative to the bulk phase, as a function of the film thickness. The resulting formula depends only on the elastic parameters, the activation volume and the diameters of the crystallites in the film layers, see eqn (32). Thus, it is of general character.

We also performed a literature survey to compile a summary of the recent experimental studies on anion $\left(\mathrm{O}^{2-}\right.$ and $\left.\mathrm{F}^{-}\right)$ transport in thin films and multilayers. The studies can be categorised by introducing the "slope factor" $s_{\sigma}$, which numerically describes the influence of the increasing phase boundary density on the conductivity. The least effect in the order of $10^{1} \mathrm{~nm}$ at maximum can be found for systems consisting of extrinsic ionic conductors. Higher effects above $10^{1} \mathrm{~nm}$ up to about $10^{5} \mathrm{~nm}$ at maximum can be observed if surface transport is included. Systems consisting of intrinsic conductors range in the order of $10^{3} \mathrm{~nm}$. We like to note that the apparently small strain effects in category (i) may be of greater practical significance than space charge effects in category (iii). Space charge effects will only be observed in systems with small intrinsic conductivity, where the resulting conductivity can still be much lower than in extrinsic, strongly doped electrolytes. Thus from a practical point of view, strain effects can be important in improving electrolytes with high conductivity.

Strain or structural effects are probably the origin of the modified interface transport properties for systems consisting 
of extrinsic ionic conductors. In the case of pure strain effects the observed magnitudes are in the predicted range of the model. Due to the typical elastic properties of ceramic oxides and structural limitations for coherency strain, the strain effect will only affect interface transport properties less than a factor of 10 . However, a factor of 10 is a significant increase for an electrolyte which already has a high conductivity. However, as already pointed out above, grain boundaries in strained epitaxial films will also influence the ionic conductivity - mostly in the direction of a lower conductivity. The effective total conductivity will then depend on the balance between grain boundary and strain effects. Maximal strain effects will be found in thin films with large columnar grains, where the density of grain boundaries is low and strain relaxation is not very effective. This is a highly important result, and we conclude that real "strain design" of solid electrolytes from the first requires advanced thin film deposition techniques with controlled growth of wide area epitaxial films with coarse grains.

In own studies, YSZ/rare earth metal oxide multilayers with coherent interfaces and various mismatches and thus interface strain were used. The experimentally observed dependence of the transport properties (relative to the bulk) vs. layer thickness can be fitted to the model, only using literature data for the elastic parameters and activation volume. The only fit parameter is the (average) diameter of the film crystallites. The values obtained from fitting are in accordance with the values determined by TEM. The model is able to explain on a semiqualitative level the observed effects on interface transport.

The results from the conductivity measurements on the YSZ/ $\mathrm{Lu}_{2} \mathrm{O}_{3}$ multilayer samples scatter considerably. The most important difference compared to the $\mathrm{YSZ} / \mathrm{Y}_{2} \mathrm{O}_{3^{-}}$and $\mathrm{YSZ} / \mathrm{Sc}_{2} \mathrm{O}_{3}$ multilayers is a less well defined texture/orientation of the film crystallites. This will cause additional and different types of grain boundaries between the crystallites in a layer, which may also change the ionic transport coefficient to a different extent.

To verify the influence of interface strain, still more experimental data for ionic conduction and diffusion in multilayer systems with well defined geometry and texture are required. In addition to the transport measurements also an X-ray diffraction analysis on the directional strain fields in the layers has to be performed. The functional course of the measured (average) strain perpendicular and parallel to the phase boundaries in the solid electrolyte and the insulator phase as a function of the layer thickness can be used to support the basic assumptions of the model.

\subsection{Limitations of a simple strain based model}

It comes out that strain effects will only influence the ionic transport in a layered system (of solid electrolytes with a high concentration of mobile charge carriers) at maximum by one order of magnitude. Grain boundaries in the bulk of the ionconducting layers influence the total conductivity in the same order of magnitude due to their blocking effect on ionic transport. Thus, only samples with similar grain boundary density and texture may be safely compared to explore the role of strain effects. The (average) diameter of the crystallites is in addition to the layer thickness also a crucial parameter determining the influence of the strain effects on ionic transport. According to the model, the (average) diameter of the crystallites directly controls the strain relaxation in the interface regions and thus the measured initial slopes.

Our model is based on isotropic mechanical behaviour. Only an isostatic pressure is assumed to introduce the pressure dependence of the ionic transport coefficients. This can only be a first order approach to demonstrate the fundamental effect of strain on interface transport. It can be improved by using the full (anisotropic) compliance tensors. The results would depend on the space group, the orientation of the film crystallites and the current direction in the interface plane. As well, the partial derivatives of the free activation enthalpy with respect to the stress components have to be considered separately.

\section{Acknowledgements}

We like to thank Dietrich Hesse from the Max-Planck-Institut für Mikrostrukturphysik (Halle/Saale, Germany) for the TEM investigations and many helpful discussions. The author CK likes to thank the DFG for financial support to proceed with the experimental studies. The author JJ acknowledges support by the project STORE-E (LOEWE program funded by the State of Hessen) and LaMa (Laboratory of Materials Science).

\section{References}

1 L. Blum, H.-P. Buchkremer, S. Gross, A. Gubner, L. G. J. de Haart, H. Nabielek, W. J. Quadakkers, U. Reisgen, M. J. Smith, R. Steinberger-Wilckens, R. W. Steinbrech, F. Tietz and I. C. Vinke, Fuel Cells, 2007, 7, 204-210.

2 S. D. Souza, S. J. Visco and L. C. D. Jonghe, Solid State Ionics, 1997, 98, 57-61.

3 S. Hui, J. Roller, S. Yick, X. Zhang, C. Decès-Petit, Y. Xie, R. Maric and D. Ghosh, J. Power Sources, 2007, 172, 493-502.

4 J. G. Swallow, W. H. Woodford, Y. C. Q. Lu, J. J. Kim, D. Chen, Y.-M. Chiang, W. C. Carter, B. Yildiz, H. L. Tuller and K. J. V. Vliet, J. Electroceram., 2014, 32, 3-27.

5 J. Garcia-Barriocanal, A. Rivera-Calzada, M. Varela, Z. Sefrioui, E. Iborra, C. Leon, S. J. Pennycook and J. Santamaria, Science, 2008, 321, 676-680.

6 C. Korte, A. Peters, D. Hesse, N. Zakharov and J. Janek, Phys. Chem. Chem. Phys., 2008, 10, 4623-4635.

7 N. Schichtel, C. Korte, D. Hesse and J. Janek, Phys. Chem. Chem. Phys., 2009, 11, 3043-3048.

8 M. Sillassen, P. Eklund, N. Pryds, E. Johnson, U. Helmersson and J. Bøttiger, Adv. Funct. Mater., 2010, 20, 2071-2076.

9 N. Schichtel, C. Korte, D. Hesse, N. Zakharov, B. Butz, D. Gerthsen and J. Janek, Phys. Chem. Chem. Phys., 2010, 12, 14596-14608.

10 K. M. Kant, V. Esposito and N. Pryds, Appl. Phys. Lett., 2012, 100, 033105.

11 B. Li, J. Zhang, T. Kaspar, V. Shutthanandan, R. C. Ewing and J. Lian, Phys. Chem. Chem. Phys., 2013, 15, 1296. 
12 H. Aydin, C. Korte, M. Rohnke and J. Janek, Phys. Chem. Chem. Phys., 2013, 15, 1944-1955.

13 H. Aydin, C. Korte and J. Janek, Sci. Technol. Adv. Mater., 2013, 14, 035007.

14 J. Jiang, X. Hu, W. Shen, C. Ni and J. L. Hertz, Appl. Phys. Lett., 2013, 102, 143901.

15 W. Shen, J. Jiang and J. L. Hertz, RSC Adv., 2014, 4, 21625-21630.

16 A. Kushima and B. Yildiz, J. Mater. Chem., 2010, 20, 4809-4819.

17 J. A. Hirschfeld and H. Lustfeld, Phys. Rev. B: Condens. Matter Mater. Phys., 2011, 84, 224308.

18 G. Dezanneau, J. Hermet and B. Dupé, Int. J. Hydrogen Energy, 2012, 37, 8081-8086.

19 R. A. de Souza, A. Ramadan and S. Hörner, Energy Environ. Sci., 2012, 5, 5445.

20 J. A. Hirschfeld and H. Lustfeld, Phys. Rev. B: Condens. Matter Mater. Phys., 2014, 89, 014305.

21 B. Yildiz, MRS Bull., 2014, 39, 147-156.

22 D. S. Aidhy, Y. Zhang and W. J. Weber, J. Phys. Chem. C, 2014, 118, 4207-4212.

23 M. Burbano, D. Marrocchelli and G. W. Watson, J. Electroceram., 2014, 32, 28-36.

24 C. Korte, N. Schichtel, D. Hesse and J. Janek, Monatsh. Chem., 2009, 140, 1069-1080.

25 J. Santiso and M. Burriel, J. Solid State Electrochem., 2011, 15, 985-1006.

26 J. L. M. Rupp, Solid State Ionics, 2012, 207, 1-13.

27 S. Schweiger, M. Kubicek, F. Messerschmitt, C. Murer and J. L. M. Rupp, ACS Nano, 2014, 8, 5032-5048.

28 J. Laurencin, G. Delette, F. Lefebvre-Joud and M. Dupeux, J. Eur. Ceram. Soc., 2008, 28, 1857-1869.

29 A. Hughes and S. Badwal, Solid State Ionics, 1991, 46, 265-274.

30 G. S. A. M. Theunissen, A. J. A. Winnubst and A. J. Burggraaf, J. Mater. Sci., 1992, 27, 5057-5066.

31 S. P. S. Badwal, F. T. Ciacchi, S. Rajendran and J. Drennan, Solid State Ionics, 1998, 109, 167-186.

32 M. de Ridder, R. G. van Welzenis, A. W. D. van der Gon, H. H. Brongersma, S. Wulff, W.-F. Chu and W. Weppner, J. Appl. Phys., 2002, 92, 3056-3064.

33 C. Chung, K. V. Hansen and M. Mogensen, Ceram. Eng. Sci. Proc., 2004, 25, 407.

34 J. Maier, J. Electrochem. Soc., 1987, 134, 1524-1535.

35 J. Maier, Solid State Ionics, 1994, 70-71, 43-51.

36 S. Jiang and J. B. Wagner, J. Phys. Chem. Solids, 1995, 56, 1101-1111.

37 S. Jiang and J. B. Wagner, J. Phys. Chem. Solids, 1995, 56, 1113-1124.

38 J. Maier, Solid State Ionics, 1995, 75, 139-145.

39 J. Maier, Prog. Solid State Chem., 1995, 23, 171-263.

40 H. Tuller, Solid State Ionics, 2000, 131, 143-157.

41 J. Maier, Solid State Ionics, 2000, 131, 13-22.

42 J. Maier, J. Eur. Ceram. Soc., 2004, 24, 1251-1257.

43 J. Maier, Solid State Ionics, 2004, 175, 7-12.

44 I. Kosacki, C. M. Rouleau, P. F. Becher, J. Bentley and D. H. Lowndes, Electrochem. Solid-State Lett., 2004, 7, A459-A461.
45 I. Kosacki, C. M. Rouleau, P. F. Becher, J. Bentley and D. H. Lowndes, Solid State Ionics, 2005, 176, 1319-1326.

46 S. Azad, O. A. Marina, C. M. Wang, L. Saraf, V. Shutthanandan, D. E. McCready, A. El-Azab, J. E. Jaffe, M. H. Engelhard, C. H. F. Peden and S. Thevuthasan, Appl. Phys. Lett., 2005, 86, 131906.

47 A. Karthikeyan, C.-L. Chang and S. Ramanathan, Appl. Phys. Lett., 2006, 89, 183116.

48 A. Peters, C. Korte, D. Hesse, N. Zakharov and J. Janek, Solid State Ionics, 2007, 178, 67-76.

49 A. Karthikeyan and S. Ramanathan, J. Appl. Phys., 2008, 104, 124314.

50 S. Sanna, V. Esposito, A. Tebano, S. Licoccia, E. Traversa and G. Balestrino, Small, 2010, 6, 1863-1867.

51 D. Pergolesi, E. Fabbri, S. N. Cook, V. Roddatis, E. Traversa and J. A. Kilner, ACS Nano, 2012, 6, 10524-10534.

52 D. Lubben and F. A. Modine, J. Appl. Phys., 1996, 80, 5150-5157.

53 N. Sata, K. Eberman, K. Eberl and J. Maier, Nature, 2000, 408, 946-949.

54 S. Furusawa and T. Kawaguchi, J. Phys. Soc. Jpn., 2001, 70, 3585-3590.

55 N. Sata, N. Y. Jin-Philipp, K. Eberl and J. Maier, Solid State Ionics, 2002, 154-155, 497-502.

56 F. Shin-ichi, S. Satoshi, S. Kaduhiro and T. Hitoshi, Solid State Ionics, 2004, 167, 325-329.

57 N. Y. Jin-Phillipp, N. Sata, J. Maier, C. Scheu, K. Hahn, M. Kelsch and M. Rühle, J. Chem. Phys., 2004, 120, 2375-2381.

58 N. Kuwata, N. Sata, T. Tsurui and H. Yugami, Jpn. J. Appl. Phys., Part 1, 2005, 44, 8613-8618.

59 X. Guo and J. Maier, Adv. Funct. Mater., 2009, 19, 96.

60 N. Schichtel, C. Korte, D. Hesse, N. Zakharov and J. Janek, Phys. Chem. Chem. Phys., 2015, to be published.

61 H.-R. Kim, J.-C. Kim, K.-R. Lee, H.-I. Ji, H.-W. Lee, J.-H. Lee and J.-W. Son, Phys. Chem. Chem. Phys., 2011, 13, 6133-6137.

62 X. Guo, Scr. Mater., 2011, 65, 96-101.

63 A. Cavallaro, M. Burriel, J. Roqueta, A. Apostolidis, A. Bernardi, A. Tarancón, R. Srinivasan, S. N. Cook, H. L. Fraser, J. A. Kilner, D. W. McComb and J. Santiso, Solid State Ionics, 2010, 181, 592-601.

64 A. P. Sutton and R. W. Balluffi, Interfaces in Crystalline Materials, Clarendon Press, 1995.

65 M. Murakami, Thin Solid Films, 1980, 69, 253-267.

66 A. Fischer, Cryst. Res. Technol., 1983, 18, 1415-1422.

67 S. Luryi and E. Suhir, Appl. Phys. Lett., 1986, 49, 140-142.

68 T. Stoica and L. Vescan, J. Cryst. Growth, 1993, 131, 32-40.

69 A. Fischer, H. Kühne, B. Roos and H. Richter, Semicond. Sci. Technol., 1994, 9, 2195-2198.

70 D. Hull and D. J. Bacon, Introduction to Dislocations, Butterworth-Heinemann, Oxford, 3rd edn, 1997, vol. 37.

71 J. Kreisel, M. C. Weber, N. Dix, F. Sánchez, P. A. Thomas and J. Fontcuberta, Adv. Funct. Mater., 2012, 22, 5044-5049.

72 M. Beyeler and D. Lazarus, Z. Naturforsch., A: Phys. Sci., 1971, 26, 291-299. 
73 E. T. Park and J.-H. Park, Proceedings of the 3rd International Meeting of Pacific Rim Ceramic Societies, Kyungju, Korea, 1998.

74 J. Oberschmidt and D. Lazarus, Phys. Rev. B: Condens. Matter Mater. Phys., 1980, 21, 5813-5822.

75 J. Oberschmidt and D. Lazarus, Phys. Rev. B: Condens. Matter Mater. Phys., 1980, 21, 2952-2961.

76 J. Oberschmidt and D. Lazarus, Phys. Rev. B: Condens. Matter Mater. Phys., 1980, 21, 5823-5834.

77 S. Matar, J. Réau, G. Demazeau, C. Lucat, J. Portier and P. Hagenmuller, Solid State Commun., 1980, 35, 681-684.

78 P. Hagenmuller, J.-M. Réau, C. Lucat, S. Matar and G. Villeneuve, Solid State Ionics, 1981, 3/4, 341-345.

79 D. R. Figueroa, J. J. Fontanella, M. C. Wintersgil, A. V. Chadwick and C. G. Andeen, J. Phys. C: Solid State Phys., 1984, 17, 4399-4411.

80 C. Varotsos, M. Lazaridou and P. Varotsos, Phys. Rev. B: Condens. Matter Mater. Phys., 1985, 32, 2634-2635.

81 I. V. Murin, O. V. Glumov, W. Gunsser and M. Karus, Radiat. Eff. Defects Solids, 1995, 137, 251-254.

82 M. Beyeler and D. Lazarus, Solid State Commun., 1969, 7, 1487-1489.

83 N. G. Pace, G. A. Saunders, Z. Sümengen and J. S. Thorp, J. Mater. Sci., 1969, 4, 1106-1110.

84 H. M. Kandil, J. D. Greiner and J. F. Smith, J. Am. Ceram. Soc., 1984, 67, 341-346.

85 R. P. Ingel and D. Lewis III, J. Am. Ceram. Soc., 1988, 71, 265-271.

86 J. W. Adams, R. Ruh and K. S. Mazdiyasni, J. Am. Ceram. Soc., 1997, 80, 903-908.

87 A. Selçuk and A. Atkinson, J. Eur. Ceram. Soc., 1997, 17, 1523-1532.

88 S. Giraud and J. Canel, J. Eur. Ceram. Soc., 2008, 28, 77-83.

89 T. Kushi, K. Sato, A. Unemoto, K. Amezawa and T. Kawada, ECS Trans., 2009, 25, 1673-1677.

90 T. Kushi, K. Sato, A. Unemoto, S. Hashimoto, K. Amezawa and T. Kawada, J. Power Sources, 2011, 196, 7989-7993.

91 S. Hart, J. Wallis and I. Sigalas, Physica B, 1986, 139 \& 140, 183-186.

92 D. Pergolesi, M. Fronzi, E. Fabbri, A. Tebano and E. Traversa, Mater. Renewable Sustainable Energy, 2013, 2, 1-9.

93 W. Baukal and R. Scheidegger, Ber. Dtsch. Keram. Ges., 1968, 45, 610-616.
94 M. Moringa and J. Cohen, Acta Crystallogr., Sect. A: Found. Crystallogr., 1979, 35, 789-795.

95 M. Yashima, S. Sakasi, M. Kakihana, Y. Yamaguchi, H. Arashi and M. Yoshimura, Acta Crystallogr., Sect. B: Struct. Sci., 1994, 50, 663-672.

96 M. Paton and E. N. Maslen, Acta Crystallogr., 1965, 19, 307-310.

97 H. O'Conner and T. Valentine, Acta Crystallogr., Sect. B: Struct. Sci., 1969, 25, 2140-2144.

98 H. Ishibashi, K. Shimomoto and K. Nakahigashi, J. Phys. Chem. Solids, 1994, 55, 809-814.

99 S. Geller, P. Romo and J. P. Remeika, Z. Kristallogr., 1967, 124, 136-142.

100 H. Bommer, Z. Anorg. Allg. Chem., 1939, 241, 273-280.

101 D. H. Templeton and C. H. Dauben, J. Am. Chem. Soc., 1954, 76, 5237-5239.

102 K. Song, H. Schmid, V. Sro, E. Gilardi, G. Gregori, K. Du, J. Maier and P. A. van Aken, APL Mater., 2014, 2, 032104.

103 T. Kiguchi, T. J. Konno, H. Funakubo, O. Sakurai and K. Shinozaki, J. Ceram. Soc. Jpn., 2014, 121, 72-77.

104 J. L. M. Rupp, B. Scherrer, N. Schäuble and L. J. Gauckler, Adv. Funct. Mater., 2010, 20, 2807-2814.

105 J. L. M. Rupp, E. Fabbri, D. Marrocchelli, J.-W. Han, D. Chen, E. Traversa, H. L. Tuller and B. Yildiz, Adv. Funct. Mater., 2014, 24, 1562-1574.

106 J. A. Thornton, Annu. Rev. Mater. Sci., 1977, 7, 239-260.

107 R. Messier, A. P. Giri and R. A. Roy, J. Vac. Sci. Technol., A, 1984, 2, 500-503.

108 A. N. Papathanassiou and J. Grammatikakis, Phys. Rev. B: Condens. Matter Mater. Phys., 1996, 53, 16247-16251.

109 A. Kossoy, Y. Feldman, E. Wachtel, I. Lubomirsky and J. Maier, Adv. Funct. Mater., 2007, 17, 2393-2398.

110 A. Kossoy, Y. Feldman, R. Korobko, E. Wachtel, I. Lubomirsky and J. Maier, Adv. Funct. Mater., 2009, 19, 634-641.

111 J. A. V. Orman, C. Li and K. L. Crispin, Phys. Earth Planet. Inter., 2009, 172, 34-42.

112 S. Estradé, J. M. Rebled, J. Arbiol, F. Peiró, I. C. Infante, G. Herranz, F. Sánchez, J. Fontcuberta, R. Córdoba, B. G. Mendis and A. L. Bleloch, Appl. Phys. Lett., 2009, 95, 072507.

113 Y. Zhu, A. Chen, H. Zhou, W. Zhang, J. Narayan, J. L. MacManus-Driscoll, Q. Jia and H. Wang, APL Mater., 2013, 1, 050702 . 